\title{
Invertible Polynomial Representation for Private Set Operations
}

\author{
Jung Hee Cheon, Hyunsook Hong, and Hyung Tae Lee \\ CHRI \& Department of Mathematical Sciences, Seoul National University \\ 1 Gwanak-ro, Gwanak-gu, Seoul 151-747, Korea \\ \{jhcheon, hongsuk07,htsm1138\}@snu.ac.kr
}

\begin{abstract}
In many private set operations, a set is represented by a polynomial over a ring $\mathbb{Z}_{\sigma}$ for a composite integer $\sigma$, where $\mathbb{Z}_{\sigma}$ is the message space of some additive homomorphic encryption. While it is useful for implementing set operations with polynomial additions and multiplications, a polynomial representation has a limitation due to the hardness of polynomial factorization over $\mathbb{Z}_{\sigma}$. That is, it is hard to recover a corresponding set from a resulting polynomial over $\mathbb{Z}_{\sigma}$ if $\sigma$ is not a prime.

In this paper, we propose a new representation of a set by a polynomial over $\mathbb{Z}_{\sigma}$, in which $\sigma$ is a composite integer with known factorization but a corresponding set can be efficiently recovered from a polynomial except negligible probability in the security parameter. Note that $\mathbb{Z}_{\sigma}[x]$ is not a unique factorization domain, so a polynomial may be written as a product of linear factors in several ways. To exclude irrelevant linear factors, we introduce a special encoding function which supports early abort strategy. As a result, our representation can be efficiently inverted by computing all the linear factors of a polynomial in $\mathbb{Z}_{\sigma}[x]$ whose roots locate in the image of the encoding function. When we consider group decryption as in most private set operation protocols, inverting polynomial representations should be done without a single party possessing the secret of the utilized additive homomorphic encryption. This is very hard for Paillier's encryption whose message space is $\mathbb{Z}_{N}$ with unknown factorization of $N$. Instead, we detour this problem by using Naccache-Stern encryption with message space $\mathbb{Z}_{\sigma}$ where $\sigma$ is a smooth integer with public factorization.

As an application of our representation, we obtain a constant round privacy-preserving set union protocol. Our construction improves the complexity than the previous without an honest majority. It can be also used for a constant round multi-set union protocol and a private set intersection protocol even when decryptors do not possess a superset of the resulting set.
\end{abstract}

Keywords: Polynomial representation, Polynomial factorization, Root finding, Privacy-preserving set union

\section{Introduction}

Privacy-preserving set operations (PPSO) are to compute set operations of participants' dataset without revealing any information other than the result. There have been many proposals to construct PPSO protocols with various techniques such as general MPC [13,1], polynomial representations $[9,17,10,24,14]$, pseudorandom functions [15], and blind RSA signatures $[7,6]$. While the last two techniques are hard to be generalized into multi-party protocols, polynomial representations combining with additive homomorphic encryption (AHE) schemes enable us to have multi-party PPSO protocols for various operations including set intersection [17, 10, 24], (over-)threshold set union [17], element reduction [17] and so on. Among these constructions, set intersection protocols run in constant rounds, but others run in linear of the number of participants.

Let us focus on privacy-preserving set union protocols. There are two obstacles to construct constant round privacy-preserving multi-party set union protocols based on polynomial representations with AHE schemes. First, in the polynomial representations set union corresponds to polynomial multiplication, which is not supported by an AHE scheme in constant rounds. Second, to recover the union set from the resulting polynomial, we need a root finding algorithm of a polynomial over $\mathbb{Z}_{\sigma}$, where $\mathbb{Z}_{\sigma}$ is the message space of the AHE scheme.

Recently, Seo et al. [25] proposed a constant round set union protocol based on a novel approach in which a set is represented as a rational function using the reversed Laurent series. 
In their protocol, each participant takes part in the protocol with a rational function whose poles consist of the elements of his set and at the end of the protocol he obtains a rational function whose poles correspond to the set union. Then each participant recovers the denominator of the rational function using the extended Euclidean algorithm and finds the roots of the denominator. Since each rational function is summed up to the resulting function after encrypted under an AHE scheme, the first obstacle is easily overcome.

However, a root finding is still problematic on the message space $\mathbb{Z}_{\sigma}$ of the AHE schemes. Since the message space has unknown order [22] or is not a unique factorization domain (UFD) [21, $23,3]$ in the current efficient AHE schemes, there is no proper polynomial factorization or root finding algorithm working on the message space. To avoid this obstacle, the authors in [25] utilized a secret sharing scheme. However, it requires computational and communicational costs heavier than the previous and requires an honest majority for security since their protocol exploits a secret sharing scheme to support privacy-preserving multiplications in constant rounds.

Our Contribution Let $\sigma=\prod_{i=1}^{\bar{\ell}} q_{i}$ for distinct primes $q_{i}$, which is larger than the size of the universe of set elements. We propose a new representation of a set by a polynomial over $\mathbb{Z}_{\sigma}$, in which a corresponding set can be efficiently recovered from a polynomial except negligible probability when the factorization of $\sigma$ is given.

For a given polynomial $f(x)=\prod_{i=1}^{d}\left(x-s_{i}\right) \in \mathbb{Z}_{\sigma}[x]$, if the factorization of $\sigma$ is given, one can obtain all roots of $f$ in $\mathbb{Z}_{q_{i}}$ for each $i$ by exploiting a polynomial factorization algorithm over a finite field $\mathbb{Z}_{q_{i}}$ [31]. By reassembling the roots of $f$ in $\mathbb{Z}_{\sigma}$ using the Chinese Remainder Theorem (CRT), we can obtain all the candidates. However, the number of candidates amounts to $d^{\bar{\ell}}$, which is exponential in the size of the universe.

We introduce a special encoding function $\iota$ to exclude irrelevant candidates efficiently. For a polynomial $f=\prod_{i=1}^{d}\left(x-\iota\left(s_{i}\right)\right) \in \mathbb{Z}_{\sigma}[x]$, our encoding function aborts most irrelevant candidates without $d^{\bar{\ell}}$ CRT computations, by giving a certain relation among roots of $f$ in $\mathbb{Z}_{q_{j}}[x]$ and roots of $f$ in $\mathbb{Z}_{q_{j+1}}[x]$. As a result, our encoding function enables us to efficiently recover all the roots of $f$ with negligible failure probability if they are in the image of $\iota$.

Table 1. Comparison with Previous Set-Union Protocols

\begin{tabular}{|c||c|c|c|c|}
\hline HBC & Rounds & Communication Cost & Computational Cost & \# of Honest Party \\
\hline \hline$[17]$ & $O(n)$ & $O\left(n^{3} k \tau_{N}\right)$ & $O\left(n^{4} k^{2} \tau_{N} \rho_{N}\right)$ & $\geq 1$ \\
\hline$[10]$ & $O(n)$ & $O\left(n^{2} k \tau_{N}\right)$ & $O\left(n^{2} k^{2} \tau_{N} \rho_{N}\right)$ & $\geq 1$ \\
\hline$[25]$ & $O(1)$ & $O\left(n^{4} k^{2} \tau_{p^{\prime}}\right)$ & $O\left(n^{5} k^{2} \rho_{p^{\prime}}\right)$ & $\geq 1$ \\
\hline \hline Ours & $O(1)$ & $O\left(n^{3} k \tau_{N}\right)$ & $O\left(n^{3} k^{2} \tau_{N} \rho_{N}\right)$ & $\geq 1$ \\
\hline Malicious & Rounds & Communication Cost & Computational Cost & \# of Honest Party \\
\hline \hline$[10]$ & $O(n)$ & $O\left(\left(n^{2} k^{2}+n^{3} k\right) \tau_{N}\right)$ & $O\left(n^{2} k^{2} \tau_{N} \rho_{N}\right)$ & $\geq n / 2$ \\
\hline$[25]$ & $O(1)$ & $O\left(n^{4} k^{2} \tau_{p}\right)$ & $O\left(n^{5} k^{2} \tau_{p} \rho_{p}\right)$ & $\geq 1$ \\
\hline \hline Ours & $O(1)$ & $O\left(n^{3} k^{2} \tau_{N}\right)$ & $O\left(n^{3} k^{2} \tau_{N} \rho_{N}\right)$ & \\
\hline
\end{tabular}

$n$ : the number of participants, $k$ : the maximum size of sets

$\tau_{N}, \tau_{p^{\prime}}, \tau_{p}$ : the size of modulus $N$ for Paillier encryption scheme or NS encryption scheme, the size $p^{\prime}$ of representing domain, the order $p$ of a cyclic group for Pedersen commitment scheme, respectively

$\rho_{N}, \rho_{p^{\prime}}, \rho_{p}$ : modular multiplication cost of modulus $N$ for Paillier encryption scheme or NS encryption scheme, $p^{\prime}$ for the size of representing domain, $p$ for the order of a cyclic group for Pedersen commitment scheme, respectively

As an application of our new representation, combining with Naccache-Stern (NS) AHE scheme which is the factorization of $\sigma$ is public, we obtain an efficient constant round privacypreserving set union protocol without an honest majority. In Table $1^{1}$, we compare our set union protocols with the previous main results $[10,17,25]$.

\footnotetext{
${ }^{1}$ Note that the communication and computational complexities in Table 1 of [25] are for one participant.
} 
Remark that we can easily extend our set union protocol to a multi-set union protocol by encoding the same elements differently. We describe details in Section 4.3. The resulting multiset union protocol is little bit slower than the previous result [14], but the public key size of the utilized encryption in our protocol is $O(1)$, while that of the previous is $O(d)$ for the size $d$ of the multi-set union.

We also consider transforming previous privacy-preserving set intersection protocol in [17] into a protocol even when decryptors do not possess a superset of the resulting set except the universe.

Related Work There have been a few researches to construct a privacy-preserving multi-party set and multi-set union protocol. Kissner and Song [17] provided multi-party set and multi-set union protocols in the honest-but-curious case. Frikken [10] and Sang and Shen [24] presented more efficient multi-party set union protocols and multi-set union protocols in the malicious case, respectively. However, these protocols exploit a mix-net protocol [11] instead of a root finding algorithm, which runs in linear rounds in the number of corrupted players, and hence it cannot run in constant rounds. Blanton and Aguiar [2] presented efficient privacy-preserving set and multi-set union protocols based on a secret sharing technique and these can be parallelized, but still run in linear rounds.

Recently, Seo et al. [25] proposed a constant round multi-party set union protocol by representing elements in a set as poles of a rational function. However, their constructions hire a secret sharing technique for supporting privacy-preserving multiplications in constant rounds, thus requires an honest majority assumption for security and computational and communicational complexity heavier than the previous.

In case of privacy-preserving multi-set union protocols, Hong et al. [14] proposed a protocol based on ElGamal encryption schemes defined over an extension field $\mathbb{F}_{q^{\kappa}}$ where $\kappa$ is larger than $d$ for the cardinality $d$ of the resulting multi-set. However, it suffers from the public key size of the utilized encryption since the extension degree $\kappa$ of the extension field has to be larger than $d$ and hence the public key size is $O(d)$.

Outline of the Paper In Section 2 we look into some components of our privacy-preserving set union protocol, polynomial factorization algorithm, polynomial representation, and AHE schemes. We provide our new polynomial representation that enables us to uniquely factorize a polynomial satisfying some criteria in Section 3. Our constant round privacy-preserving set union protocols are presented in Section 4. Some supplying materials including analysis of our representation and the set union protocol for the malicious case, are given in Appendix.

\section{Preliminaries}

In this section, we look into polynomial representations of a set for PPSO protocols and introduce efficient AHE schemes utilized in PPSO protocols to support polynomial operations between encrypted polynomials. Then, we briefly look into root finding algorithms over finite fields and message spaces of AHE schemes for applying to recover a set from a polynomial in the polynomial representation.

\subsection{Basic Definitions and Notations}

Throughout the paper, let $\mathcal{U}$ be the universe, $n$ be the number of participants in the protocol, and $k$ be the maximum size of participants' datasets $S_{i}$ 's. Also, $d$ denotes the size of (multi-)set union among participants' datasets in the protocol.

Let $R[x]$ be a set of polynomials defined over a ring $R$ and $R(x)$ be a set of rational functions defined over R, i.e., $R[x]=\left\{f(x) \mid f(x)=\sum_{i=0}^{\operatorname{deg} f} f[i] x^{i}\right.$ and $f[i] \in R$ for all $\left.i\right\}$ and $R(x)=$ $\left\{\frac{f(x)}{g(x)} \mid f(x), g(x) \in R[x], g(x) \neq 0\right\}$. For a polynomial $f \in R[x]$, we denote the coefficient of $x^{i}$ in 
a polynomial $f$ by $f[i]$, i.e., $f(x)=\sum_{i=0}^{\operatorname{deg} f} f[i] x^{i} \in R[x]$. For a polynomial $f(x)=\sum_{i=0}^{\operatorname{deg} f} f[i] x^{i} \in$ $\mathbb{Z}_{\sigma}[x]$ and a factor $q$ of $\sigma, f \bmod q$ denotes a polynomial $\sum_{i=0}^{\operatorname{deg} f}(f[i] \bmod q) x^{i} \in \mathbb{Z}_{q}[x]$.

We also define a negligible function as follows: a function $g: \mathbb{N} \rightarrow \mathbb{R}$ is negligible if for every positive polynomial $\mu(\lambda)$, there exists an integer $N$ such that $g(\lambda)<1 / \mu(\lambda)$ for all $\lambda>N$.

\subsection{Polynomial Representation of a Set}

Let $R$ be a commutative ring with unity and $S$ be a subset of $R$. We may represent a set $S$ by a polynomial or a rational function over $R$.

Polynomial Representation In some previous works $[9,17,10,14,25]$, a set $S$ can be represented by a polynomial $f_{S}(x) \in R[x]$ whose roots are the elements of $S$. That is,

$$
f_{S}(x):=\prod_{s_{i} \in S}\left(x-s_{i}\right) .
$$

This representation gives the following relation:

$$
f_{S}(x)+f_{S^{\prime}}(x)=\operatorname{gcd}\left(f_{S}(x), f_{S^{\prime}}(x)\right) \cdot u(x)
$$

for some polynomial $u(x) \in R[x]$ and hence the roots of a polynomial $f_{S}(x)+f_{S^{\prime}}(x)$ are the elements of $S \cap S^{\prime}$ with overwhelming probability. Also, the roots of $f_{S}(x) \cdot f_{S^{\prime}}(x)$ are the elements of $S \cup S^{\prime}$ as multi-sets.

Rational function Representation Recently, Seo et al. [25] introduced a novel representation of a set $S \subset R$ by a rational function $F_{S}$ over $R$ whose poles consist of the elements of $S$. That is,

$$
F_{S}(x):=\frac{1}{\prod_{s_{i} \in S}\left(x-s_{i}\right)}=\frac{1}{f_{S}(x)} .
$$

This representation provides the following relation:

$$
F_{S}(x)+F_{S^{\prime}}(x)=\frac{f_{S}(x)+f_{S^{\prime}}(x)}{f_{S}(x) \cdot f_{S^{\prime}}(x)}=\frac{\operatorname{gcd}\left(f_{S}(x), f_{S^{\prime}}(x)\right) \cdot u(x)}{f_{S}(x) \cdot f_{S^{\prime}}(x)}=\frac{u(x)}{\operatorname{lcm}\left(f_{S}(x), f_{S^{\prime}}(x)\right)}
$$

for some polynomial $u(x) \in R[x]$ which is relatively prime to $\operatorname{lcm}\left(f_{S}(x), f_{S^{\prime}}(x)\right)$ with overwhelming probability. Hence the poles of $F_{S}(x)+F_{S^{\prime}}(x)$ are exactly the roots of $\operatorname{lcm}\left(f_{S}(x), f_{S^{\prime}}(x)\right)$, which are the elements of $S \cup S^{\prime}$ as sets, not multi-sets, if $u(x)$ and $\operatorname{lcm}\left(f_{S}(x), f_{S^{\prime}}(x)\right)$ have no common roots. This rational function is represented again by an infinite formal power series, so called a Reversed Laurent Series (RLS), in [25].

\subsection{Additive Homomorphic Encryption}

Let us consider a commutative ring $R$ with unity and a $R$-module $G$ where $r \cdot g:=g^{r}$ for $r \in R$ and $g \in G$. Let Enc pk $: R \rightarrow G$ be a public key encryption under the public key pk. We can define a public key encryption for a polynomial $f=\sum_{i=0}^{\operatorname{deg} f} f[i] x^{i} \in R[x]$ as follows:

$$
\mathcal{E}_{\mathrm{pk}}(f):=\sum_{i=0}^{\operatorname{deg} f} \operatorname{Enc}_{\mathrm{pk}}(f[i]) x^{i} .
$$

Assume Enc pk $_{\text {has }}$ an additive homomorphic property such that

$$
\operatorname{Enc}_{\mathrm{pk}}(a+b)=\operatorname{Enc}_{\mathrm{pk}}(a) \operatorname{Enc}_{\mathrm{pk}}(b), \quad \operatorname{Enc}_{\mathrm{pk}}(a b)=\operatorname{Enc}_{\mathrm{pk}}(a)^{b}
$$

for $a, b \in R$. Then given two polynomials $f=\sum_{i=0}^{\operatorname{deg} f} f[i] x^{i}$ and $g=\sum_{i=0}^{\operatorname{deg} g} g[i] x^{i}$ in $R[x]$, we can induce homomorphic properties of $\mathcal{E}$ as follows: 
- Polynomial addition: Given $\mathcal{E}_{\mathrm{pk}}(f)$ and $\mathcal{E}_{\mathrm{pk}}(g)$, it is possible to compute $\mathcal{E}_{\mathrm{pk}}(f+g)$ by calculating $\operatorname{Enc}_{\mathrm{pk}}((f+g)[i])=\operatorname{Enc}_{\mathrm{pk}}(f[i]) \operatorname{Enc}_{\mathrm{pk}}(g[i])$ for all $0 \leq i \leq \max \{\operatorname{deg} f, \operatorname{deg} g\}$ where $f+g=\sum_{i=0}^{\max \{\operatorname{deg} f, \operatorname{deg} g\}}(f+g)[i] x^{i}$.

- Polynomial multiplication: Given $\mathcal{E}_{\mathrm{pk}}(f)$ and $g$, it is possible to compute $\mathcal{E}_{\mathrm{pk}}(f g)$ by calculating $\operatorname{Enc}_{\mathrm{pk}}((f g)[\ell])=\prod_{i+j=\ell} \operatorname{Enc}_{\mathrm{pk}}(f[i])^{g[j]}$ for all $0 \leq \ell \leq \operatorname{deg} f+\operatorname{deg} g$ where $f g=$ $\sum_{\ell=0}^{\operatorname{deg} f+\operatorname{deg} g}(f g)[\ell] x^{\ell}$.

There have been several efficient AHE schemes [21-23,3]: Under the assumption that factoring $N=p^{2} q$ is hard, Okamoto and Uchiyama [22] proposed a scheme with $R=\mathbb{Z}_{p}$ and $G=\mathbb{Z}_{N}$, in which the order $p$ of the message space $R$ is hidden. With the decisional composite residuosity assumption, Paillier [23] scheme and Camenisch and Shoup scheme [3] have $R=\mathbb{Z}_{N}$ and $G=\mathbb{Z}_{N^{2}}$ for $N=p q$, in which the size of message spaces is a hard-to-factor composite integer $N$. Naccache and Stern [21] proposed a scheme with $R=\mathbb{Z}_{\sigma}$ and $G=\mathbb{Z}_{N}$ under the higher residuosity assumption, where $N=p q$ is a hard-to-factor integer and $\sigma$ is a product of small primes dividing $\phi(N)$ for Euler's totient function $\phi$.

In the above schemes, it is hard to find the roots of a polynomial in $R[x]$ without knowing a secret key. For the second case, in fact, Shamir [27] showed that to find a root of a polynomial $f(x)=\prod_{i=1}^{d}\left(x-s_{i}\right) \in \mathbb{Z}_{N}[x]$ is equivalent to factor $N$. While, in the NS scheme, it may be possible to compute some roots of a polynomial in $\mathbb{Z}_{\sigma}[x]$ since the factorization of $\sigma$ is public. But $\mathbb{Z}_{\sigma}[x]$ is not a UFD and hence the number of roots of a polynomial $f \in \mathbb{Z}_{\sigma}[x]$ can be larger than $\operatorname{deg} f$. In fact, if $f(x)=\prod_{i=1}^{d}\left(x-s_{i}\right) \in R[x]$, then the number of candidates of roots of the polynomial $f$ is $d^{\bar{\ell}}$ where $\bar{\ell}$ is the number of prime factors of $\sigma$. We will use the NS scheme by presenting a method to efficiently recover all the roots of a polynomial $f \in \mathbb{Z}_{\sigma}[x]$ satisfying some criteria.

\subsection{Root Finding Algorithms}

When $R$ is a finite field $\mathbb{F}_{q}$, we have several efficient root finding algorithms in $R[x]$. As an instance, using a square-free decomposition and the Cantor-Zassenhaus algorithm [32, Section 14.4], a polynomial of degree $d$ over a field $\mathbb{F}_{q}$ is factored in $\widetilde{O}\left(d^{2} \log q\right)$ field operations. Recently, it has been improved using fast arithmetic into $O\left(d^{1.5+o(1)}\right)$ field operations by Umans [31]. However, as mentioned above, there is no efficient AHE scheme whose message space is a finite field $\mathbb{F}_{q}$ with public $q$.

Consider $R=\mathbb{Z}_{\sigma}$, a message space of the NS encryption scheme for $\sigma=\prod_{j=1}^{\bar{\ell}} q_{j}$ with distinct public primes $q_{j}$ 's. Let $f \in \mathbb{Z}_{\sigma}[x]$ be a polynomial of degree $d$, which is a product of $d$ linear factors $\left(x-s_{i}\right)$ 's. Since $\mathbb{Z}_{\sigma}[x]$ is not a UFD, it is still very hard to recover the exact $\left(x-s_{i}\right)$ 's from the polynomial $f$. However, since the factors of $\sigma$ are known, one can apply Umans' polynomial factorization algorithm to find all roots of $f \bmod q_{j}$ with $\widetilde{O}\left(d^{1.5}\right)$ field operations in $\mathbb{Z}_{q_{j}}$ for each $j$. Then one performs the CRT computations, which takes $O\left(\log ^{2} \sigma\right)$ bit operations for each candidate of roots. Since each polynomial $f \bmod q_{j}$ has about $d$ distinct roots, there exist about $d^{\bar{\ell}}$ candidates of roots of $f$ over $\mathbb{Z}_{\sigma}$. Hence the total complexity becomes $\widetilde{O}\left(\bar{\ell} d^{1.5} \log ^{2} q+d^{\bar{\ell}} \log ^{2} \sigma\right)$ bit operations, where $q:=\max _{j}\left\{q_{j}\right\}$. However, one still can not determine the exact $s_{i}$ 's since it has no criterion to distinguish the exact $s_{i}$ 's from candidates.

In this paper, we can recover all the exact roots of $f$ satisfying some criteria in $O\left(\bar{\ell} d^{1.5} \log ^{2} q\right)$ bit operations using early abort technique. The details are in Section 3.

\section{Invertible Polynomial Representation}

Let $f_{S}(x)=\prod_{s_{i} \in S}\left(x-s_{i}\right) \in \mathbb{Z}_{\sigma}[x]$ be a polynomial for a set $S \subseteq \mathbb{Z}_{\sigma}$ and a composite $\sigma=\prod_{j=1}^{\bar{\ell}} q_{j}$ with distinct primes $q_{j}$ 's. Since $\mathbb{Z}_{\sigma}[x]$ is not a UFD, it is impossible to recover the exact $S$ from 
the polynomial $f_{S}$ in almost all cases. In this section, we provide our new polynomial representation that enables us to efficiently recover the exact corresponding set from the polynomial represented by our suggestion.

Before describing our polynomial representation, we assume that the resulting set union of cardinality $d$ is randomly chosen in $\mathcal{U}_{(d)}$, the set of subsets of cardinality $d$ of the universe $\mathcal{U}$. In general, since elements of a participant's dataset may not be random in the universe $\mathcal{U}$, the resulting set union may also not be random in the set $\mathcal{U}_{(d)}$. However, one can efficiently make that the resulting set union satisfies this assumption using a pseudorandom permutation. Also, in practice, one can utilize a block cipher such as the DES encryption instead of a pseudorandom permutation [12, Section 3.7]. For example, a participant encrypts his element $s^{\prime}$ so that $s=$ $\mathrm{DES}_{K_{3}}\left(\mathrm{DES}_{K_{2}}\left(\mathrm{DES}_{K_{1}}\left(s^{\prime}\right)\right)\right)$ where $K_{1}, K_{2}$ and $K_{3}$ are public and randomly chosen in the key space of the DES encryption. Then a participant obtains the original message $s^{\prime}$ by computing $s^{\prime}=\operatorname{DES}_{K_{1}}^{-1}\left(\operatorname{DES}_{K_{2}}^{-1}\left(\operatorname{DES}_{K_{3}}^{-1}(s)\right)\right)$ after recovering a root $s$ from the resulting polynomial.

Parameter Setting Let us explain parameters for our polynomial representation and PPSO protocols. First, set the bit size of the modulus $N$ of the NS encryption scheme by considering a security parameter $\lambda$. For the given universe $\mathcal{U}$ and the maximum size of the resulting set union $d$ (here, $d=n k$ for the number $n$ of participants and the maximum size $k$ of participants' datasets), let $d_{0}=\max \{d,\lceil\log N\rceil\}$ and set $\tau=\frac{1}{3}\left(\log d+2 \log d_{0}\right)$. This setting comes from the computational complexity analysis of our set union protocol and the value $\tau$ will influence the bit size of prime factors of $\sigma$ and the size of the message space of the NS encryption scheme. See Section 4.2 for details.

Set the parameter $\ell$ and $\alpha$ so that $\ell$ is the smallest positive integer such that $\mathcal{U} \subseteq\{0,1\}^{3 \tau \alpha \ell}$ for some rational number $0<\alpha<1$ satisfying $3 \alpha \tau$ and $3(1-\alpha) \tau$ are integers. Note that the proper size of $\alpha$ is $\frac{1}{3}$ for optimal complexity of our protocol. The details will be presented in Appendix A. Then, set the proper size $\bar{\ell}$ larger than $\ell$ and let $\ell^{\prime}=\bar{\ell}-\ell$. The analysis of the proper size of $\bar{\ell}$ will be discussed at the end of Section 3.1. Choose $\bar{\ell}(3 \tau+1)$-bit distinct primes $q_{j}$ 's and set $\sigma=\prod_{j=1}^{\bar{\ell}} q_{j}$. Note that the size of the message space of the NS encryption scheme is less than $\frac{N}{4}$ for its security [21]. Hence, the parameters have to be satisfied the condition $\sigma<\frac{N}{4}$ and so $\bar{\ell}<\frac{\lfloor\log N\rfloor-2}{3 \tau}$. Also, we assume that $\bar{\ell}$ is smaller than $d$ for optimal complexity of our proposed protocol. In summary, the parameter $\bar{\ell}$ is smaller than $\min \left\{d, \frac{\lfloor\log N\rfloor-2}{3 \tau}\right\}$.

Now, we are ready to describe our new polynomial representation. Focus on the fact that the factorization of $\sigma$ is public in the NS encryption scheme. Using this fact, given a polynomial $f=\prod_{i=1}^{d}\left(x-s_{i}\right) \in \mathbb{Z}_{\sigma}[x]$ for a set $S=\left\{s_{1}, \ldots, s_{d}\right\}$, one can obtain all roots of $f \bmod q_{j}$ for each $j$ by applying Umans' polynomial factorization algorithm over a finite field $\mathbb{Z}_{q_{j}}$. To recover $S$, one can perform CRT computation for obtaining less than $d^{\bar{\ell}}$ candidates of roots of $f$ over $\mathbb{Z}_{\sigma}$. In general, however, the number of roots of $f$ over $\mathbb{Z}_{\sigma}$ is larger than $\operatorname{deg} f$ and there is no criteria to determine the exact set $S$. To remove irrelevant roots which are not in $S$, we give some relations among all roots of polynomials $f \bmod q_{j}$ 's by providing an encoding function.

Before describing our solution, we look into an easy way to give a relation among all roots of polynomial $f \bmod q_{j}$ for all $j$. However, it is not efficient to recover a set from a polynomial.

Encoding with a tag To give relations among all roots of polynomials $f$ mod $q_{i}$ 's, we may consider to insert the same value depending on the element, called a tag, into an element part in $\mathbb{Z}_{q_{j}}$ 's. For example, let $h:\{0,1\}^{*} \rightarrow\{0,1\}^{3(1-\alpha) \tau}$ be an uniform hash function for a positive rational number $0<\alpha<1$ such that $3 \alpha \tau$ and $3(1-\alpha) \tau$ are to be integers. For a dataset $S \subseteq \mathbb{Z}_{\sigma}$, parse $s_{i} \in S$ into $\bar{\ell}$ blocks $s_{i, 1}, \ldots, s_{i, \bar{\ell}}$ of $(3 \alpha \tau)$-bit so that $s_{i}=s_{i, 1}\|\cdots\| s_{i, \bar{\ell}}$. Consider a function $\iota^{\prime}: \mathcal{U} \subseteq\{0,1\}^{3 \alpha \tau \bar{\ell}} \rightarrow \mathbb{Z}_{\sigma}$, in which $\iota^{\prime}\left(s_{i}\right)$ is the unique element in $\mathbb{Z}_{\sigma}$ satisfying 
$\iota^{\prime}\left(s_{i}\right) \equiv s_{i, j} \| h\left(s_{i}\right) \bmod q_{j}$ for $1 \leq j \leq \bar{\ell}^{2}$ Then we represent a set $S$ as a polynomial $f_{S}(x)=$ $\prod_{s_{i} \in S}\left(x-\iota^{\prime}\left(s_{i}\right)\right) \in \mathbb{Z}_{\sigma}[x]$.

Then one can reduce the number of candidates by checking a tag $h\left(s_{i}\right)$ when one gets all roots over $\mathbb{Z}_{q_{j}}$ 's. However, when a collision occurs, say $h\left(s_{i}\right)=h\left(s_{j}\right)$ with $s_{i} \neq s_{j}$, one has to check the hash value of $2^{\bar{\ell}}$ elements which are possible combinations of $\left(s_{i, 1}, s_{j, 1}\right), \cdots,\left(s_{i, \bar{\ell}}, s_{j, \bar{\ell}}\right)$. The probability ${ }^{3}$ that at least one collision occur among $d$ hash values $h\left(s_{i}\right)$ 's is lower bounded by $\frac{d^{2}}{2^{1+3 \tau(1-\alpha)}}$ which is not negligible even for small $\alpha$. Moreover, the expected computation becomes $\Omega\left(2^{\bar{\ell}}\right)$.

\subsection{Our Polynomial Representation}

Now, we present our polynomial representation for supporting to recover a set from a polynomial over $\mathbb{Z}_{\sigma}$ represented by our suggestion. Take $\alpha=\frac{1}{3}$, i.e., $\mathcal{U} \subseteq\{0,1\}^{\tau \ell}$ for optimization. If $\alpha \neq \frac{1}{3}$, the expected computation is in polynomial time only when the size of the universe is restricted. Details about the proper size of $\alpha$ is in Appendix A.

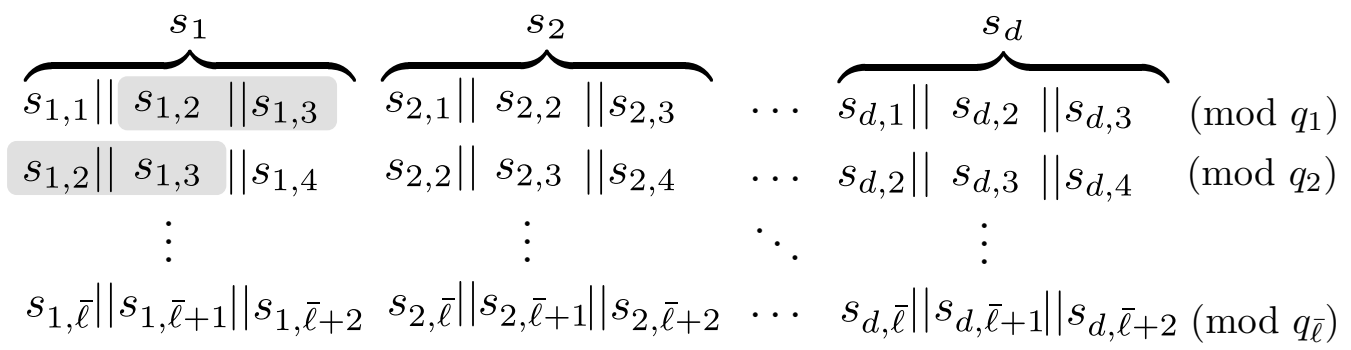

Fig. 1. Our Encoding Function $\iota$

Encoding by Repetition Let $h:\{0,1\}^{*} \rightarrow\{0,1\}^{2 \tau}$ and $h_{j}:\{0,1\}^{*} \rightarrow\{0,1\}^{\tau}$ be uniform hash functions for $1 \leq j \leq \ell^{\prime}$. Parse a message $s_{i} \in \mathcal{U} \subseteq\{0,1\}^{\tau \ell}$ into $\ell$ blocks $s_{i, 1}, \ldots, s_{i, \ell}$ of $\tau$-bit so that $s_{i}=s_{i, 1}\|\cdots\| s_{i, \ell}$. Let $s_{i, \ell+j}=h_{j}\left(s_{i}\right)$ for $1 \leq j \leq \ell^{\prime}$ and parse $h\left(s_{i}\right)$ into two blocks $s_{i, \bar{\ell}+1}$ and $s_{i, \bar{\ell}+2}$ of $\tau$-bit. Set $\bar{\ell}=\ell+\ell^{\prime}$. We define our encoding function $\iota: \mathcal{U} \subseteq\{0,1\}^{\tau \ell} \rightarrow \mathbb{Z}_{\sigma}$, in which $\iota\left(s_{i}\right)$ is the unique element in $\mathbb{Z}_{\sigma}$ satisfying $\iota\left(s_{i}\right) \equiv s_{i, j}\left\|s_{i, j+1}\right\| s_{i, j+2} \bmod q_{j}$ for $1 \leq j \leq \bar{\ell}$. Then a set $S$ is represented as a polynomial $f_{S}(x)=\prod_{s_{i} \in S}\left(x-\iota\left(s_{i}\right)\right) \in \mathbb{Z}_{\sigma}[x]$.

Decoding Phase Denote by $s_{j}^{(i)}:=\iota\left(s_{i}\right) \bmod q_{j}$ for each message $s_{i}=s_{i, 1}\|\cdots\| s_{i, \ell}$. For $1 \leq$

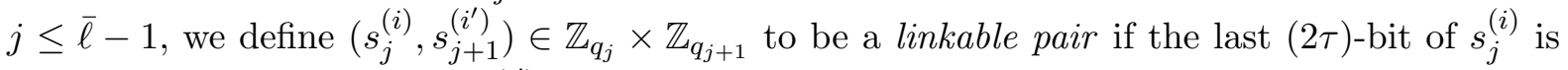
equal to the first $(2 \tau)$-bit of $s_{j+1}^{\left(i^{\prime}\right)}$, i.e., $s_{i, j+1}\left\|s_{i, j+2}=s_{i^{\prime}, j+1}\right\| s_{i^{\prime}, j+2}$. Inductively, we also define $\left(s_{1}^{\left(i_{1}\right)}, \cdots, s_{j+1}^{\left(i_{j+1}\right)}\right) \in \mathbb{Z}_{q_{1}} \times \cdots \times \mathbb{Z}_{q_{j+1}}$ to be a linkable pair if $\left(s_{1}^{\left(i_{1}\right)}, \cdots, s_{j}^{\left(i_{j}\right)}\right)$ and $\left(s_{j}^{\left(i_{j}\right)}, s_{j+1}^{\left(i_{j+1}\right)}\right)$ are linkable pairs.

Let $\iota\left(s_{i}\right)$ and $\iota\left(s_{i^{\prime}}\right)$ be images of elements $s_{i}$ and $s_{i^{\prime}}$ of the function $\iota$ with $s_{i} \neq s_{i^{\prime}}$. We can easily check the following properties:

$-\left(s_{1}^{(i)}, \cdots, s_{j+1}^{(i)}\right)$ is always a linkable pair.

\footnotetext{
${ }^{2}$ By notation abuse, throughout this paper if necessary, we regard a bit string as the corresponding integer via some converting function.

${ }^{3} \operatorname{Pr}$ [At least one collision occur among $d$ hash values $h\left(s_{i}\right)$ 's. $] \leq 1-\left(1-\frac{1}{2^{3(1-\alpha) \tau}}\right) \cdots\left(1-\frac{d-1}{2^{3(1-\alpha) \tau}}\right) \approx 1-$ $\exp \left(-\prod_{i=1}^{d-1} \frac{i}{2^{3(1-\alpha) \tau}}\right) \approx \frac{d^{2}}{2 \cdot 2^{3(1-\alpha) \tau}}$.
} 


$$
\begin{aligned}
& s_{1}^{\left(i_{1}\right)}=s_{i_{1}, 1}\left\|s_{i_{1}, 2}\right\| s_{i_{1}, 3} \\
& s_{2}^{\left(i_{2}\right)}=\quad s_{i_{2}, 2}\left\|s_{i_{2}, 3}\right\| s_{i_{2}, 4} \\
& s_{3}^{\left(i_{3}\right)}=\quad \quad s_{i_{3}, 3}\left\|s_{i_{3}, 4}\right\| s_{i_{3}, 5} \\
& \Rightarrow\left(s_{1}^{\left(i_{1}\right)}, s_{2}^{\left(i_{2}\right)}, s_{3}^{\left(i_{3}\right)}\right) \text { is a linkable pair. }
\end{aligned}
$$

Fig. 2. Linkable Pair

- When $s_{i}$ and $s_{i^{\prime}}$ are uniformly chosen strings from $\{0,1\}^{\tau \ell}$,

$$
\operatorname{Pr}\left[\left(s_{j}^{(i)}, s_{j}^{\left(i^{\prime}\right)}\right) \text { is a linkable pair }\right]=\operatorname{Pr}\left[s_{i, j+1}\left\|s_{i, j+2}=s_{i^{\prime}, j+1}\right\| s_{i^{\prime}, j+2}\right]=\frac{1}{2^{2 \tau}}
$$

for a fixed $1 \leq j \leq \bar{\ell}$

At decoding phase, when a polynomial $f(x)=\prod_{i=1}^{d}\left(x-\iota\left(s_{i}\right)\right) \in \mathbb{Z}_{\sigma}[x]$ is given, we perform two phases to find the correct $d$ roots of the polynomial $f(x)$. In the first stage, one computes all the roots $\left\{s_{j}^{(1)}, \cdots, s_{j}^{(d)}\right\}$ over $\mathbb{Z}_{q_{j}}[x]$ for each $j$. For each $j$ sequentially from 1 to $\bar{\ell}-1$, we find all the linkable pairs among $\left\{s_{j}^{(1)}, \cdots, s_{j}^{(d)}\right\}$ and $\left\{s_{j+1}^{(1)}, \cdots, s_{j+1}^{(d)}\right\}$ by checking whether the last $(2 \tau)$-bit of $s_{j}^{(i)}$ and the first $(2 \tau)$-bit of $s_{j+1}^{\left(i^{\prime}\right)}$ are the same. It can be done by $d^{2}$ comparisons or $O(d \log d)$ computations using sorting and determining algorithms.

After $\bar{\ell}-1$ steps, we obtain $d^{\prime}$ number of linkable pairs of $\bar{\ell}$-tuple, which are candidates of roots of the polynomial $f$ and elements of the set. It includes the $d$ elements corresponding to $\iota\left(s_{1}\right), \ldots, \iota\left(s_{d}\right)$. If $d^{\prime}$ is much larger than $d$, it can be a burden. However, we can show that the expected value of $d^{\prime}$ is at most $3 d$ in Theorem 1. See Section 3.2.

After obtaining $d^{\prime}$ linkable pairs of $\bar{\ell}$-tuple, in the second phase, we check whether each pair belongs to the image of $\iota$ with the following equalities:

$$
\begin{aligned}
s_{i, \ell+j} & =h_{j}\left(s_{i}\right) \quad \text { for all } 1 \leq j \leq \ell^{\prime}, \\
s_{i, \bar{\ell}+1} \| s_{i, \bar{\ell}+2} & =h\left(s_{i}\right) .
\end{aligned}
$$

The linkable pairs of $\bar{\ell}$-tuple, corresponding to $\iota\left(s_{i}\right)$ for some $i$ clearly satisfies the above equations. However, for a random $\bar{\ell}$-tuple in $\mathbb{Z}_{q_{1}} \times \cdots \times \mathbb{Z}_{q_{\bar{\ell}}}$, the probability that it satisfies the relation (2) is about $\frac{1}{2^{2 \ell^{\prime}}}$ and the probability that it satisfies the relation (3) is about $\frac{1}{2^{2 \tau}}$ under the assumption that $h$ and $h_{j}$ 's are uniform hash functions. Hence, the expected number of wrong $\bar{\ell}$-tuples passing both phases is less than $d \times \frac{1}{2^{\tau\left(2+\ell^{\prime}\right)}}$. It is less than $2^{-\lambda}$ for a security parameter $\lambda$ if we take the parameter $\ell^{\prime}$ to satisfy

$$
\ell^{\prime}>\frac{3(\lambda+\log d)}{\log d+2 \log d_{0}}-2 .
$$

For example, when $\lambda=80$ and $d \approx d_{0} \approx 2^{10}$, then $\ell^{\prime}$ is about 8 . Therefore, one can recover a set from the given polynomial represented by our suggestion without negligible failure probability in the security parameter.

Computational Complexity Let us count the computational cost of our representation. The encoding phase consists of two steps: (1) the CRT computation per each element to obtain a value of the encoding function $\iota$ and (2) the polynomial expansion. The first step requires $O\left(d \log ^{2} \sigma\right)$ bit operations for $d$ elements and the second step requires $O\left(d^{2}\right)$ multiplications. Hence, the complexity for the encoding phase is $O\left(d^{2}\right)$ multiplications.

The decoding phase may be divided into three steps: (1) finding roots of a polynomial $f$ in $\mathbb{Z}_{q_{j}}$ for each $j,(2)$ finding all linkable pairs of length $\bar{\ell}$, and (3) checking the equations (2) 
and (3). These steps require $O\left(\bar{\ell} d^{1.5}\right)$ multiplications, $O(\bar{\ell} d \log d)$ bit operations, and $O\left(\ell^{\prime} d\right)$ hash computations, respectively. Hence, the complexity for the decoding phase is dominated by $O\left(\bar{\ell} d^{1.5}\right)$ multiplications.

\subsection{The Expected Number of Linkable Pairs}

In this subsection, we analyze the expected number of linkable pairs of $\bar{\ell}$-tuple when we recover a set from a polynomial of degree $d$, represented by our suggestion.

A set of $\kappa$ elements $s_{j}^{(1)}, \cdots, s_{j}^{(\kappa)} \in \mathbb{Z}_{q_{j}}$ is called a $\kappa$-collision if their last $2 \tau$-bits are the same. Since $\left(s_{j-1}^{(i)}, s_{j}^{(i)}\right)$ is trivially a linkable pair for $1 \leq i \leq \kappa, \kappa$-collision causes at least $\kappa$ linkable pairs. Assume that $S=\left\{s_{1}, \ldots, s_{d}\right\}$ is a uniformly and randomly chosen set in the set of subsets of cardinality $d$ of the set $\{0,1\}^{\tau \ell}$ and $h$ and $h_{j}$ 's utilized in the encoding function $\iota$ are uniform hash functions. Then, we easily obtain the following observations, which are evidences of the expected number of linkable pairs of $\bar{\ell}$-tuple is not large.

1. The probability that at least one 2 -collision occurs in $\mathbb{Z}_{q_{j}}$ is less than $\frac{1}{2}$ by the birthday paradox.

2. The probability that at least one $\kappa$-collision occurs for $\kappa \geq 3$ in $\mathbb{Z}_{q_{j}}$ is at most $\frac{1}{4 d} \approx \frac{1}{2^{(2+\tau)}}$ of the probability that at least one $(\kappa-1)$-collision occurs from [30, Theorem 2].

3 . The $\kappa$-collision in $\mathbb{Z}_{q_{j}}$ yields $\kappa^{2}$ more candidates of roots of the polynomial $f$, not $2^{\kappa}$ candidates. More concretely, assume that $\kappa$-collision $\left\{s_{j}^{(1)}, \cdots, s_{j}^{(\kappa)}\right\}$ occurs. Then $s_{j}^{(1)}$ can be combined with $\kappa$ candidates $\left\{s_{j+1}^{(1)}, \cdots, s_{j+1}^{(\kappa)}\right\}$. Hence $\kappa^{2}$ linkable pairs are generated.

The expected number of 2-collision in $\mathbb{Z}_{q_{j}}$ for all $j$ is roughly $\frac{\bar{\ell}}{2}$ and the expected number of $\kappa$-collision in $\mathbb{Z}_{q_{j}}$ for $\kappa \geq 3$ is negligible. Theorem 1 gives a rigorous analysis of the upper bound of the expected number of linkable pairs of $\bar{\ell}$-tuple.

Theorem 1. Assume that $S=\left\{s_{1}, \ldots, s_{d}\right\}$ is a uniformly and randomly chosen set in the set of subsets of cardinality $d$ of the set $\{0,1\}^{\tau \ell}$. Define an encoding function $\iota:\{0,1\}^{\tau \ell} \rightarrow \mathbb{Z}_{\sigma}$ so that $\iota\left(s_{i}\right)$ is the unique element in $\mathbb{Z}_{\sigma}$ satisfying $\iota\left(s_{i}\right) \equiv s_{i, j}\left\|s_{i, j+1}\right\| s_{i, j+2} \bmod q_{j}$ for all $1 \leq j \leq \bar{\ell}$ when $s_{i}=s_{i, 1}\|\ldots\| s_{i, \ell}$ and $s_{i, j}$ 's are $\tau$-bit. Assume $h$ and $h_{j}$ 's utilized in the encoding function $\iota$ are uniform hash functions. Then the expected number of linkable pairs of $\bar{\ell}$-tuple is at most $3 d$ for a polynomial $f_{S}=\prod_{s_{i} \in S}\left(x-s_{i}\right)$.

Proof. Let $\mathrm{E}_{j}$ be the expected number of linkable pairs of $j$-tuple in $\mathbb{Z}_{q_{1}} \times \cdots \times \mathbb{Z}_{q_{j}}$ for $j \geq 2$. For $1 \leq j \leq j^{\prime} \leq \bar{\ell}$, let $\mathrm{S}_{j^{\prime}-j+1}\left(i_{j}, \ldots, i_{j^{\prime}}\right)$ be the event that $\left(s_{j}^{\left(i_{j}\right)}, \ldots, s_{j^{\prime}}^{\left(i_{j^{\prime}}\right)}\right)$ is a linkable pair. Then,

$$
\begin{aligned}
\mathrm{E}_{2} & =\sum_{i_{1}, i_{2} \in\{1, \ldots, d\}} 1 \cdot \operatorname{Pr}\left[\mathrm{S}_{2}\left(i_{1}, i_{2}\right)\right] \\
& =\sum_{i_{1}, i_{2} \in\{1, \ldots, d\}} \operatorname{Pr}\left[\mathrm{S}_{2}\left(i_{1}, i_{2}\right) \wedge\left(i_{1}=i_{2}\right)\right]+\sum_{i_{1}, i_{2} \in\{1, \ldots, d\}} \operatorname{Pr}\left[\mathrm{S}_{2}\left(i_{1}, i_{2}\right) \wedge\left(i_{1} \neq i_{2}\right)\right] \\
& =\sum_{i_{1} \in\{1, \ldots, d\}} \operatorname{Pr}\left[\mathrm{S}_{2}\left(i_{1}, i_{1}\right)\right]+\sum_{i_{1} \neq i_{2} \in\{1, \ldots, d\}} \operatorname{Pr}\left[\mathrm{S}_{2}\left(i_{1}, i_{2}\right)\right] \\
& =d+d(d-1) \frac{1}{2^{2 \tau}}=d\left(1+\frac{d-1}{2^{2 \tau}}\right)
\end{aligned}
$$

since $\operatorname{Pr}\left[\mathrm{S}_{2}\left(i_{1}, i_{1}\right)\right]=1$ for $i_{1} \in\{1, \ldots, d\}$ and $\operatorname{Pr}\left[\mathrm{S}_{2}\left(i_{1}, i_{2}\right)\right]=\frac{1}{2^{2 \tau}}$ for distinct $i_{1}, i_{2} \in\{1, \ldots, d\}$ from the equation (1).

Now, we consider the relation between $\mathrm{E}_{j}$ and $\mathrm{E}_{j+1}$. When $\left(s_{1}^{\left(i_{1}\right)}, \ldots, s_{j}^{\left(i_{j}\right)}\right)$ is a linkable pair, consider the case that $\left(s_{1}^{\left(i_{1}\right)}, \ldots, s_{j}^{\left(i_{j}\right)}, s_{j+1}^{\left(i_{j+1}\right)}\right)$ is a linkable pair. One can classify this case into the following three cases: 
1. $i_{j+1}=i_{j}$,

2. $\left(i_{j+1} \neq i_{j}\right) \wedge\left(i_{j+1}=i_{j-1}\right)$,

3. $\left(i_{j+1} \neq i_{j}\right) \wedge\left(i_{j+1} \neq i_{j-1}\right)$.

At the first case, if $i_{j+1}=i_{j}$ and $\left(s_{1}^{\left(i_{1}\right)}, \ldots, s_{j}^{\left(i_{j}\right)}\right)$ is a linkable pair, then $\left(s_{1}^{\left(i_{1}\right)}, \ldots, s_{j}^{\left(i_{j}\right)}, s_{j+1}^{\left(i_{j+1}\right)}\right)$ is always a linkable pair. Hence,

$$
\begin{aligned}
\mathrm{E}_{j+1}^{(1)} & :=\sum_{i_{1}, \ldots, i_{j+1} \in\{1, \ldots, d\}} \operatorname{Pr}\left[\mathrm{S}_{j+1}\left(i_{1}, \ldots, i_{j}, i_{j+1}\right) \wedge\left(i_{j+1}=i_{j}\right)\right] \\
& =\sum_{i_{1}, \ldots, i_{j} \in\{1, \ldots, d\}} \operatorname{Pr}\left[\mathrm{S}_{j}\left(i_{1}, \ldots, i_{j}\right)\right]=\mathrm{E}_{j} .
\end{aligned}
$$

At the second case, if $i_{j+1}=i_{j-1} \neq i_{j}$ and $\left(s_{1}^{\left(i_{1}\right)}, \ldots, s_{j}^{\left(i_{j}\right)}\right)$ is a linkable pair, then the relation $s_{i_{j-1}, j+1}=s_{i_{j}, j+1}=s_{i_{j+1}, j+1}$ is satisfied from the encoding rule of $\iota$. Hence,

$$
\begin{aligned}
\mathrm{E}_{j+1}^{(2)} & :=\sum_{i_{1}, \ldots, i_{j+1} \in\{1, \ldots, d\}} \operatorname{Pr}\left[\mathrm{S}_{j+1}\left(i_{1}, \ldots, i_{j}, i_{j+1}\right) \wedge\left(i_{j+1}=i_{j-1} \neq i_{j}\right)\right] \\
& =\sum_{\substack{i_{1}, \ldots, i_{j+1} \in\{1, \ldots, d\} \\
i_{j+1}=i_{j-1} \neq i_{j}}} \operatorname{Pr}\left[\mathrm{S}_{j}\left(i_{1}, \ldots, i_{j}\right) \wedge \mathrm{S}_{2}\left(i_{j}, i_{j+1}\right)\right] \\
& =\sum_{\substack{i_{1}, \ldots, i_{j+1} \in\{1, \ldots, d\} \\
i_{j+1}=i_{j-1} \neq i_{j}}} \operatorname{Pr}\left[\mathrm{S}_{j}\left(i_{1}, \ldots, i_{j}\right) \wedge\left(s_{i_{j}, j+1}\left\|s_{i_{j}, j+2}=s_{i_{j+1}, j+1}\right\| s_{i_{j+1}, j+2}\right)\right] \\
& =\sum_{\substack{i_{1}, \ldots, i_{j+1} \in\{1, \ldots, d\} \\
i_{j+1}=i_{j-1} \neq i_{j}}} \operatorname{Pr}\left[\mathrm{S}_{j}\left(i_{1}, \ldots, i_{j}\right) \wedge\left(s_{i_{j}, j+2}=s_{i_{j+1}, j+2}\right)\right] \\
& =\sum_{\substack{i_{1}, \ldots, i_{j+1} \in\{1, \ldots, d\} \\
i_{j+1}=i_{j-1} \neq i_{j}}} \operatorname{Pr}\left[\mathrm{S}_{j}\left(i_{1}, \ldots, i_{j}\right)\right] \operatorname{Pr}\left[s_{i_{j}, j+2}=s_{i_{j+1}, j+2}\right] \\
& =\frac{1}{2^{\tau}} \sum_{\substack{i_{1}, \ldots, i_{j+1} \in\{1, \ldots, d\} \\
i_{j+1}=i_{j-1} \neq i_{j}}} \operatorname{Pr}\left[\mathrm{S}_{j}\left(i_{1}, \ldots, i_{j}\right)\right] \\
& \leq \frac{1}{2^{\tau}} \sum_{\substack{i_{1}, \ldots, i_{j} \in\{1, \ldots, d\} \\
i_{1}}} \operatorname{Pr}\left[\mathrm{S}_{j}\left(i_{1}, \ldots, i_{j}\right)\right]=\frac{1}{2^{\tau}} \mathrm{E}_{j} .
\end{aligned}
$$

At the last case, we can obtain the following result:

$$
\begin{aligned}
\mathrm{E}_{j+1}^{(3)} & :=\sum_{\substack{i_{1}, \ldots, i_{j+1} \in\{1, \ldots, d\} \\
i_{1}, \ldots, i_{j+1} \in\{1, \ldots, d\} \\
i_{j+1} \notin\left\{i_{j-1}, i_{j}\right\}}} \operatorname{Pr}\left[\mathrm{S}_{j+1}\left(i_{1}, \ldots, i_{j}, i_{j+1}\right) \wedge\left(\left(i_{1}, \ldots, i_{j}\right) \wedge \mathrm{S}_{2}\left(i_{j}, i_{j+1}\right)\right]\right. \\
& \left.=\sum_{\substack{i_{1}, \ldots, i_{j+1} \in\{1, \ldots, d\} \\
i_{j+1} \notin\left\{i_{j-1}, i_{j}\right\}}} \operatorname{Pr}\left[\mathrm{S}_{j}\left(i_{1}, \ldots, i_{j}\right) \wedge\left(s_{i_{j}, j+1} \| s_{i_{j}, j+2}=i_{j-1}\right)\right)\right] \\
& =\sum_{\substack{i_{j+1}, j+1 \\
i_{1}, \ldots, i_{j+1} \in\{1, \ldots, d\} \\
i_{j+1} \notin\left\{i_{j-1}, i_{j}\right\}}} \operatorname{Pr}\left[\mathrm{S}_{j}\left(i_{i_{j+1}, j+2}, \ldots, i_{j}\right)\right] \operatorname{Pr}\left[s_{i_{j}, j+1}\left\|s_{i_{j}, j+2}=s_{i_{j+1}, j+1}\right\| s_{i_{j+1}, j+2}\right] \\
& \leq \frac{d-1}{2^{2 \tau}} \sum_{i_{1}, \ldots, i_{j} \in\{1, \ldots, d\}} \operatorname{Pr}\left[\mathrm{S}_{j}\left(i_{1}, \ldots, i_{j}\right)\right]=\frac{d-1}{2^{2 \tau}} \mathrm{E}_{j} .
\end{aligned}
$$


From the above results, we obtain the recurrence formula of $\mathrm{E}_{j}$ as follows:

$$
\begin{aligned}
\mathrm{E}_{j+1} & =\mathrm{E}_{j+1}^{(1)}+\mathrm{E}_{j+1}^{(2)}+\mathrm{E}_{j+1}^{(3)} \\
& \leq\left(1+\frac{1}{2^{\tau}}+\frac{d-1}{2^{2 \tau}}\right) \mathrm{E}_{j}
\end{aligned}
$$

for $j \geq 2$ and hence

$$
\mathrm{E}_{\bar{\ell}} \leq d\left(1+\frac{1}{2^{\tau}}+\frac{d-1}{2^{2 \tau}}\right)^{\bar{\ell}-1}
$$

since $\mathrm{E}_{2}=d\left(1+\frac{d-1}{2^{2 \tau}}\right) \leq d\left(1+\frac{1}{2^{\tau}}+\frac{d-1}{2^{2 \tau}}\right)$.

Now, we show that $\bar{\ell} \leq \frac{2^{2 \tau}}{2^{\tau}+d}$. From the parameter setting, it is satisfied that $\bar{\ell} \leq \min \left\{d, \frac{\lfloor\log N\rfloor-2}{3 \tau}\right\}$. When $d_{0} \geq 8 d$, it holds

$$
\min \left\{d, \frac{\lfloor\log N\rfloor-2}{3 \tau}\right\} \leq d \leq \frac{d_{0}^{1 / 3} d^{2 / 3}}{2} .
$$

Consider the case that $d_{0}<8 d$. Then, it also holds

$$
\min \left\{d, \frac{\lfloor\log N\rfloor-2}{3 \tau}\right\} \leq \frac{\lfloor\log N\rfloor-2}{3 \tau} \leq \frac{d_{0}}{3 \tau} \leq \frac{d_{0}^{1 / 3} d^{2 / 3}}{2}
$$

since $\tau \geq 3$. Hence

$$
\bar{\ell} \leq \min \left\{d, \frac{\lfloor\log N\rfloor-2}{3 \tau}\right\} \leq \frac{d_{0}^{1 / 3} d^{2 / 3}}{2} \leq \frac{\left(d_{0}^{2} d\right)^{2 / 3}}{2 d_{0}} \leq \frac{2^{2 \tau}}{2^{\tau}+d}
$$

since $2 d_{0}>2^{\tau}+d$. Therefore we obtain the following result:

$$
\mathrm{E}_{\bar{\ell}} \leq d\left(1+\frac{1}{2^{\tau}}+\frac{d-1}{2^{2 \tau}}\right)^{\bar{\ell}-1}<e d<3 d
$$

where $e \approx 2.718$ is the base of the natural logarithm. In other words, the upper bound of the expected number of linkable pairs of $\bar{\ell}$-tuple is $3 d$.

\section{Applications to Set Operations}

In this section, we present our set union protocols based on our polynomial representation described in Section 3. Our construction exploits the NS AHE scheme to encrypt a rational function whose denominator corresponds to a participant's set. For this, we generalize a reversed Laurent Series presented in [25] to work on $\mathbb{Z}_{\sigma}$ with a composite $\sigma$, which is the domain of the NS scheme.

We also explain how to modify our polynomial representation for applying to construct multi-set union protocol and a set intersection protocol in which decryptors are different from set contributors.

\subsection{Transforming Our Representation into a Rational Function using Reversed Laurent Series}

To construct constant round privacy-preserving set union protocols, we adopt rational function representations presented in [25]. In the rational function representation, each participant $\mathcal{P}_{i}$ represents his own set $S_{i}$ of elements as a rational function $\frac{1}{f_{S_{i}}}$ where $f_{S_{i}}:=\prod_{s_{j} \in S}\left(x-s_{j}\right)$. It gives the relation

$$
\frac{r_{1}}{f_{S_{1}}}+\frac{r_{2}}{f_{S_{2}}}+\cdots+\frac{r_{n}}{f_{S_{n}}}=\frac{u}{\operatorname{lcm}\left(f_{S_{1}}, f_{S_{2}}, \ldots, f_{S_{n}}\right)}
$$


for random polynomials $r_{i}$ 's and some polynomial $u$. Then each participant tries to recover $f(x)=\operatorname{lcm}\left(f_{S_{1}}, \ldots, f_{S_{n}}\right)$ from a polynomial $F(x)=\frac{u}{\operatorname{lcm}\left(f_{S_{1}}, \ldots, f_{S_{n}}\right)}$.

To represent a set as a rational function, the authors in [25] exploit a reversed Laurent series (RLS). We briefly introduce a RLS. (Refer to [25] for details.)

For a positive integer $q$, a reversed Laurent series (RLS) over $\mathbb{Z}_{q}$ is a singly infinite, formal sum of the form $f(x)=\sum_{i=-\infty}^{m} f[i] x^{i}(f[m] \neq 0)$ with an integer $m$ and $f[i] \in \mathbb{Z}_{q}$ for all $i$. We define the degree of $f$ by $m$ and it is denoted by $\operatorname{deg} f$. For a RLS $f(x)$, given $d_{1} \leq d_{2} \leq \operatorname{deg} f$, we denote $f(x)_{\left[d_{1}, d_{2}\right]}=\sum_{i=d_{1}}^{d_{2}} f[i] x^{i}$. For a rational function $f / g$ with $f, g \in \mathbb{Z}_{q}[x]$ and $g \neq 0$, we define the $R L S$ representation of a rational function $f / g$ by a reversed Laurent series of $f / g$.

When $q$ is a prime, the RLS representation has the following properties:

- The RLS representation for a given rational function is unique.

- Let $f, g$ be polynomials in $\mathbb{Z}_{q}[x]$ with $\operatorname{deg} f<\operatorname{deg} g$ and $g \neq 0$. Then there exists an algorithm [25] to compute $k(>\operatorname{deg} g)$ high-order terms of the RLS representation of $f / g$. We describe this algorithm in Algorithm 1. RationalToRLS $(f, g, k)$ denotes the output of RationalToRLS algorithm which takes polynomials $f, g$ and integer $k$.

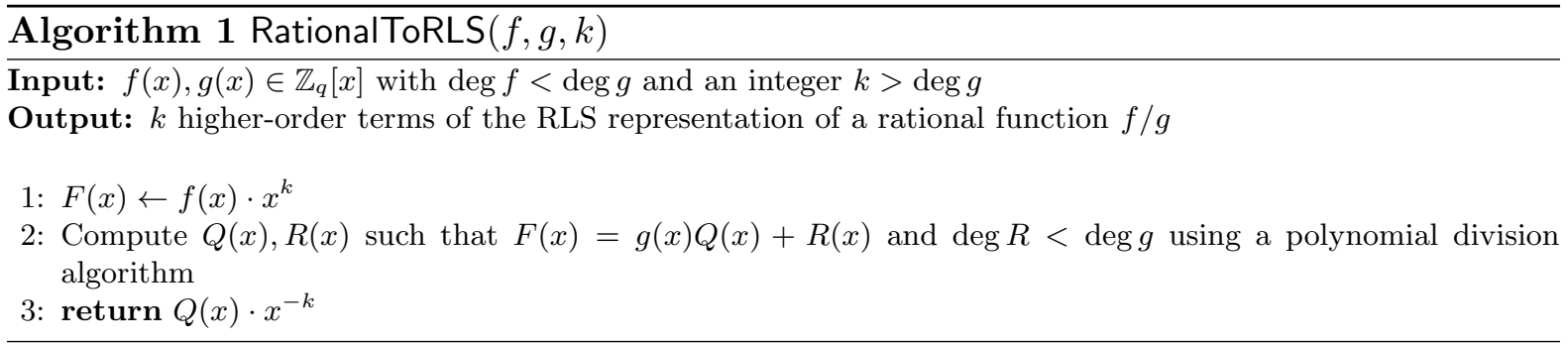

- When $2 k$ high-order terms of the RLS representation of a rational function $f / g$ such that $f, g \in \mathbb{Z}_{q}[x], g \neq 0$, and $\operatorname{deg} f<\operatorname{deg} g \leq k$ are given, there exists an efficient algorithm [29, Section 17.5.1] to recover two polynomials $v(x), u(x)$ in $\mathbb{Z}_{q}[x]$ such that $\frac{v}{u}=\frac{f}{g}$ in $\mathbb{Z}_{q}(x)$ and $\operatorname{gcd}(v, u)=1$.

In our protocol, we will represent each participant's set $S_{i}$ as our polynomial representation $f_{S_{i}}:=\prod_{s_{j} \in S_{i}}\left(x-\iota\left(s_{j}\right)\right) \in \mathbb{Z}_{\sigma}[x]$ with our encoding function $\iota$. Then we convert a rational function of $1 / f_{S_{i}}$ to its RLS over $\mathbb{Z}_{\sigma}$. Since $\mathbb{Z}_{\sigma}$ is not a Euclidean domain, one may doubt whether the RationalToRLS algorithm works on $\mathbb{Z}_{\sigma}[x]$. However, in our protocol, since the conversion requires polynomial divisions only by monic polynomials, the RationalToRLS algorithm works well on $\mathbb{Z}_{\sigma}[x]$.

After the end of interactions among participants in our protocol, each participant obtains the $2 n k$ higher-order terms of the RLS representation of a rational function $\frac{u(x)}{U(x)}$ where $U(x)=$ $\operatorname{lcm}\left(f_{S_{1}}(x), \ldots, f_{S_{n}}(x)\right)$. There is no algorithm to recover $u^{\prime}(x)$ and $U^{\prime}(x)$ in $\mathbb{Z}_{\sigma}[x]$ such that $\frac{u(x)}{U(x)}=\frac{u^{\prime}(x)}{U^{\prime}(x)}$. However, from our polynomial representation, it only requires $U^{\prime}(x) \bmod q_{j}$ for each $j$ and we can obtain $U^{\prime}(x) \bmod q_{j}$ from the RLS representation modulo $q_{j}$ by running polynomial recovering algorithm on $\mathbb{Z}_{q_{j}}[x]$ 's.

The following lemma guarantees that, in a polynomial ring $\mathbb{Z}_{\sigma}[x]$, a modular operation by a prime divisor $q$ of $\sigma$ and RationalToRLS algorithm are commutative. This will be utilized to prove the correctness of our protocol.

Lemma 1. Let $f, g$ be polynomials in $\mathbb{Z}_{\sigma}[x]$ with $\operatorname{deg} f<\operatorname{deg} g$ and $g \neq 0$. Suppose that the leading coefficient of $g$ is 1 in $\mathbb{Z}_{\sigma}$. For each prime $q$ which divides $\sigma$ and an integer $k>\operatorname{deg} g$, RationalToRLS $(f \bmod q, g \bmod q, k)=$ RationalToRLS $(f, g, k) \bmod q$. 
Proof. Let RationalToRLS $(f, g, k)=Q(x) x^{-k}$ where $x^{k} f(x)=Q(x) g(x)+R(x)$ in $\mathbb{Z}_{\sigma}[x]$ with $R=0$ or $\operatorname{deg} R<\operatorname{deg} g$. For each polynomial $p(x)$ in $\mathbb{Z}_{\sigma}[x]$, denote $p(x) \bmod q$ by $p_{q}(x)$. Then $x^{k} f_{q}(x)=Q_{q}(x) g_{q}(x)+R_{q}(x)$ in $\mathbb{Z}_{q}[x]$, where $R_{q}=0$ or $\operatorname{deg} R_{q} \leq \operatorname{deg} R<\operatorname{deg} g=$ $\operatorname{deg} g_{q}$. Since the division algorithm uniquely outputs the quotient and the remainder in $\mathbb{Z}_{q}[x]$, RationalToRLS $(f \bmod q, g \bmod q, k)=Q_{q}(x) x^{-k} \equiv Q(x) x^{-k} \bmod q$.

The following lemma gives an information on the distribution of $u_{j}(x):=u(x) \bmod q_{j}$ in our protocol which is inevitable to prove the security of our set union protocol. It guarantees that the distributions of $u_{j}(x)$ and $u(x)$ are uniformly distributed among polynomials in the set of polynomials having degree at most $\operatorname{deg}\left(\operatorname{lcm}\left(f_{S_{1}}, \ldots, f_{S_{n}}\right)\right)-1$ in $\mathbb{Z}_{q_{j}}[x]$ and $\mathbb{Z}_{\sigma}[x]$, respectively. The proof of Lemma 2 is given in [25].

Lemma 2 ([25, Lemma 1]). Let $f_{S_{1}}(x), \ldots, f_{S_{n}}(x) \in \mathbb{Z}_{q}[x]$ be polynomials of degree $k \geq$ 1 for a prime $q$. Suppose $r_{1}(x), \ldots, r_{n}(x)$ are polynomials in $\mathbb{Z}_{q}[x]$, chosen uniformly and independently in the set of polynomials of degree at most $k-1$. Let $u(x)$ be a polynomial such that

$$
\frac{u(x)}{l c m\left(f_{S_{1}}(x), \ldots, f_{S_{n}}(x)\right)}=\sum_{i=1}^{n} \frac{r_{i}(x)}{f_{S_{i}}(x)} .
$$

Then $u(x)$ is uniformly distributed among polynomials in the set of polynomials $\in \mathbb{Z}_{q}[x]$ having degree at most $\operatorname{deg}\left(\operatorname{lcm}\left(f_{S_{1}}(x), \ldots, f_{S_{n}}(x)\right)\right)-1$.

Finally, to recover the exact $U(x)=\operatorname{lcm}\left(f_{S_{1}}, \ldots, f_{S_{n}}\right)$ from the rational function $\frac{u(x)}{U(x)}$, the relation $\operatorname{gcd}(u(x), U(x))=1$ is to be satisfied. In our set union protocol, since $u_{j}(x):=$ $u(x) \bmod q_{j}$ is uniformly distributed in $\mathbb{Z}_{q_{j}}[x]$ and the expected number of roots of a random polynomial is one [19], we may expect that our RLS representation fails to output all the elements in the set union with probability $\frac{d}{q_{j}} \approx 2^{-2 \tau}$. Furthermore, it can be detected if this happens for a certain $j$, whose probability is about $1-\left(1-\frac{d}{q_{j}}\right)^{\bar{\ell}} \approx \frac{\bar{\ell} d}{q_{j}} \leq 2^{-\tau}$. It is not negligible, but small enough.

\subsection{Set Union for Honest-But-Curious Case}

Threshold Naccache-Stern Encryption For a group decryption, it requires a semantically secure, threshold NS AHE scheme in our protocol. We provide a threshold version of the NS encryption scheme in Appendix B. Our construction is based on the technique of Fouque et al. [8], which transforms the original Paillier homomorphic encryption scheme into a threshold version working from Shoup's technique [28].

Parameter Setting Let $\mathcal{U}$ be the universe, $n$ be the number of participants, and $k$ be the maximum size of participants' datasets. Let $d$ be the possible maximum size of the set union, i.e., $d=n k$. Take the bit size of $N$ by considering the security of the threshold NS AHE scheme, which is the modulus of the threshold NS AHE scheme. Put $d_{0}=\max \{d,\lceil\log N\rceil\}$ and $\tau=\frac{1}{3}\left(\log d+2 \log d_{0}\right)$. Set $\ell$ so that $\mathcal{U} \subseteq\{0,1\}^{\tau \ell}$, a proper size of $\ell^{\prime}$ so that $\ell^{\prime}$ satisfies the relation (4) and let $\bar{\ell}=\ell+\ell^{\prime}$. Note that $\bar{\ell}$ is to be smaller than $\min \left\{d, \frac{\lfloor\log N\rfloor-2}{3 \log \log N}\right\}$ since $\tau \geq \log \log N$. Generate the parameters of the threshold NS encryption scheme, including the size of message space $\sigma$, which is a product of $\bar{\ell}(3 \tau+1)$-bit distinct primes $q_{i}$ 's.

Our Set Union Protocol for Honest-But-Curious Case Our set union protocol against honestbut-curious (HBC) adversaries is described in Figure 3. In our set union protocol, each participant computes the $2 n k$ higher-order terms of the RLS representation of $F_{S_{i}}=\frac{1}{f_{S_{i}}}=$ $\frac{1}{\prod_{s_{i, j} \in S_{i}}\left(x-\iota\left(s_{i, j}\right)\right)} \in \mathbb{Z}_{\sigma}[x]$ for our encoding function $\iota$ and sends its encryption to all others. With the received encryptions of $F_{S_{j}}$ for $1 \leq j \leq n$, each participant $\mathcal{P}_{i}$ multiplies a 
Input: There are $n \geq 2 \mathrm{HBC}$ participants $\mathcal{P}_{i}$ with a private input set $S_{i} \subseteq \mathcal{U}$ of cardinality $k$. Set $d=n k$. The participants share the secret key sk, to which pk is the corresponding public key to the threshold NS AHE scheme. Let $\iota:\{0,1\}^{*} \rightarrow \mathbb{Z}_{\sigma}$ be the encoding function provided in Section 3.

Each participant $\mathcal{P}_{i}, i=1, \ldots, n$ :

1. (a) constructs the polynomial $f_{S_{i}}(x)=\prod_{s_{i, j} \in S_{i}}\left(x-\iota\left(s_{i, j}\right)\right) \in \mathbb{Z}_{\sigma}[x]$, runs RationalToRLS $\left(1, f_{S_{i}},(2 n+1) k-1\right)$ to obtain $\left(\frac{1}{f_{S_{i}}(x)}\right)_{[-(2 n+1) k+1,-k]}$, and computes $F_{S_{i}}(x)=\left(\frac{1}{f_{S_{i}}(x)}\right)_{[-(2 n+1) k+1,-k]} \cdot x^{(2 n+1) k-1}$.

(b) computes $\tilde{F}_{S_{i}}$, the encrypted polynomial of $F_{S_{i}}$, and sends $\tilde{F}_{S_{i}}$ to all other participants.

2. (a) chooses random polynomials $r_{i, j}(x) \in \mathbb{Z}_{\sigma}[x]$ of degree at most $k$ for all $1 \leq$ $j \leq n$.

(b) computes the encryption, $\tilde{\phi}_{i}$, of the polynomial $\phi_{i}(x)=\sum_{j=1}^{n} F_{S_{j}} \cdot r_{i, j}$ and sends it to all participants.

3. (a) calculates the encryption of the polynomial $F(x)=\sum_{i=1}^{n} \phi_{i}(x)$.

(b) performs a group decryption with all other players to obtain the $2 n k$ higherorder terms of $F(x)$.

4. (a) recovers a polynomial pair of $u_{j}(x)$ and $U_{j}(x)$ in $\mathbb{Z}_{q_{j}}[x]$ for all $1 \leq j \leq \bar{\ell}$ such that $\left(\frac{u_{j}(x)}{U_{j}(x)}\right)_{[-2 n k,-1]}=\left(F(x) \bmod q_{j}\right)_{[k-1,(2 n+1) k-2]} \cdot x^{-(2 n+1) k+1}$ and $\operatorname{gcd}\left(u_{j}(x), U_{j}(x)\right)=1$ in $\mathbb{Z}_{q_{j}}[x]$, using the $2 n k$ higher-order terms of $F(x)$ obtained in Step 3 (b).

(b) extracts all roots of $U_{j}(x)$ in $\mathbb{Z}_{q_{j}}[x]$ for all $j$ using a factorization algorithm.

(c) determines the set union using the encoding rule of $\iota$.

Fig. 3. PPSU-HBC protocol in the HBC case

polynomial $r_{i, j}$ using additive homomorphic property, which is a randomly chosen polynomial by the participant $\mathcal{P}_{i}$ and adds all the resulting polynomials to obtain the encryption of $\phi_{i}(x)=\sum_{j=1}^{n} F_{S_{j}} \cdot r_{i, j}$. Then, he sends the encryption of $\phi_{i}(x)$ to all others. After interactions among participants, each participant can obtain the $2 n k$ high-order term of the RLS representation of $F(x)=\sum_{i=1}^{n}\left(\sum_{j=1}^{n} \frac{1}{f_{S_{j}}} \cdot r_{i, j}\right) \in \mathbb{Z}_{\sigma}[x]$. Then each participant obtains the $2 n k$ high-order terms of the RLS representation of $F$ in $\mathbb{Z}_{\sigma}[x]$ with group decryption and recovers polynomials $u_{j}(x)$ and $U_{j}(x)$ such that $\left(\frac{u_{j}(x)}{U_{j}(x)}\right)_{[-2 n k,-1]}=\left(F(x) \bmod q_{j}\right)_{[k-1,(2 n+1) k-2]} \cdot x^{-(2 n+1) k+1}$ and $\operatorname{gcd}\left(u_{j}(x), U_{j}(x)\right)=1$ in $\mathbb{Z}_{q_{j}}[x]$ from these values. Thereafter, each participant extracts all roots of $U_{j}(x)$ over $\mathbb{Z}_{q_{j}}$ for each $j$ and recovers all elements based on criteria of our representation.

Analysis Now, we consider the correctness and privacy of our proposed protocol described in Figure 3. The following theorems guarantee the correctness and privacy of our construction in Figure 3.

Theorem 2. In the protocol described in Figure 3, every participant learns the set union of private inputs participating players, with high probability.

Proof. After Step 3 (b), all participants obtain the $2 n k$ higher-order terms of $F(x)=\sum_{i=1}^{n}\left(\sum_{j=1}^{n} \frac{1}{f_{S_{j}}} \cdot r_{i, j}\right) \in$ $\mathbb{Z}_{\sigma}[x]$ and hence they obtain the $2 n k$ higher-order terms of the RLS representation of $F(x)$ mod $q_{j}$. From these values, using polynomial recovering algorithm, they reconstruct polynomials $u_{j}(x)$ and $U_{j}(x)$ such that $\frac{u_{j}(x)}{U_{j}(x)} \equiv F_{j}(x) \bmod q_{j}$ and $\operatorname{gcd}\left(u_{j}(x), U_{j}(x)\right)=1$. From the equation (4) and Lemma $1, U_{j}=\left(\operatorname{lcm}\left(f_{S_{1}}, f_{S_{2}}, \ldots, f_{S_{n}}\right) \bmod q_{j}\right)$ with high probability. Since our polynomial representation can give the exact corresponded set with overwhelming probability, it gives $S_{1} \cup \ldots \cup S_{n}$. 
Theorem 3. Assume that the utilized additive homomorphic encryption scheme is semantically secure. Then, in our set union protocol for the HBC case described in Figure 3, any adversary $\mathcal{A}$ of colluding fewer than $n$ HBC participants learns no more information than would be gained by using the same private inputs in the ideal model with a trusted third party.

Proof. Since the utilized additive homomorphic encryption scheme is semantically secure, each participant learns only $F(x)=\sum_{j=1}^{n}\left(\sum_{i=1}^{n} r_{i, j}\right) F_{S_{j}}$ in $\mathbb{Z}_{\sigma}[x]$. All players contribute to generate the polynomial $\sum_{i=1}^{n} r_{i, j}$ and the polynomial $\sum_{i=1}^{n} r_{i, j}$ is uniformly distributed and unknown. Moreover, the resulting polynomials $u_{j}$ are uniformly distributed by Lemma 2 . Hence, no information can be recovered from the polynomial $F, U_{j}$ 's and $u_{j}$ 's, other than those given by revealing the union set.

Performance Analysis It is clear that our protocol runs in $O(1)$ rounds. Let us count the computational and communicational costs for each participant.

Step 1 (a): requires $\tilde{O}(k)$ multiplications in $\mathbb{Z}_{\sigma}$ for a polynomial expansion of degree $k$ and $O(k d)$ multiplications to run the RationalToRLS algorithm and compute $F_{S_{i}}$.

Step 1 (b): requires $O(d)$ exponentiations for $2 d$ encryptions and $O(n d)$ communication costs.

Step 2 (b): requires $O\left(d^{2}\right)$ exponentiations for computing the encryption $\tilde{\phi}_{i}:=\sum_{j=1}^{n} \tilde{F}_{S_{j}} \cdot r_{i, j}$ using additive homomorphic property and $O(n d)$ communication costs.

Step 3 (a): requires $O(n d)$ multiplications for computing $\sum_{i=1}^{n} \tilde{\phi}_{i}$.

Step $3(\mathrm{~b})$ : requires $O(d)$ exponentiations for decryption share computation for $2 d$ ciphertexts and $O\left(\bar{\ell} \sqrt{d q_{i}}\right)$ multiplications for solving $d$ DLPs for $\bar{\ell}$ groups of order $q_{j}$ 's. ${ }^{4}$ The communication cost is $O(n d)$.

Step 4 (a): requires $O\left(d^{2}\right)$ multiplications in $\mathbb{Z}_{q_{j}}$ to recover $U_{j}(x)$ using extended Euclidean algorithm for each $j$.

Step $4(\mathrm{~b})$ : requires $O\left(d^{1.5+o(1)}\right)$ multiplications in $\mathbb{Z}_{q_{j}}$ for each $j$ to factor a polynomial of degree $d$.

Step 4 (c): requires $O\left(\bar{\ell} d \log d \log q_{j}\right)$ bit operations for sorting and $O(d)$ hash computations.

Then the computational complexity is dominated by one of terms $O\left(d^{2}\right)$ exponentiations in Step 2 (b) and $O\left(\bar{\ell} \sqrt{d q_{i}}\right)$ multiplications in Step 3 (b). Since one modular exponentiation for a modulus $N$ requires $O(\log N)$ multiplications and $\bar{\ell}<\min \left\{d, \frac{\lfloor\log N\rfloor-2}{\log \log N}\right\}$, the computational complexity for each participant is dominated by $O\left(d^{2}\right)=O\left(n^{2} k^{2}\right)$ exponentiations in $\mathbb{Z}_{N}$ and the total complexity is $O\left(n^{3} k^{2}\right)$ exponentiations in $\mathbb{Z}_{N}$. The total communication cost for our protocol is $O\left(n^{2} d\right)=O\left(n^{3} k\right)(\log N)$-bit elements.

For the malicious case, we can obtain the set union protocol using techniques in [17] and [25]. Refer to Appendix C.1 for the details in the malicious case.

\subsection{Extend to Multi-set Union Protocol}

We can easily extend our set union protocol to a multi-set union protocol by modifying our encoding function. Assume that each participant $\mathcal{P}_{i}$ has a multi-set $S_{i} \subseteq \mathcal{U}$ for the known universe $\mathcal{U} \subseteq\{0,1\}^{\tau \ell}$. Define a function $\eta: \mathcal{U} \rightarrow \mathcal{U}^{\prime \prime} \subseteq\{0,1\}^{\tau\left(\ell+\ell^{\prime \prime}\right)}$ by $\bar{\eta}(s)=s \| r$ where $r$ is a randomly chosen element in $\{0,1\}^{\tau \ell^{\prime \prime}}$. Then each participant takes part in our set union protocol with a set $\left\{\eta\left(s_{1}\right), \ldots, \eta\left(s_{k}\right)\right\}$ as his set instead of $\left\{s_{1}, \ldots, s_{k}\right\}$. For the same messages $s_{1}$ and $s_{2}$, if $\eta\left(s_{1}\right)$ is different from $\eta\left(s_{2}\right)$, one can obtain $\eta\left(s_{1}\right)$ and $\eta\left(s_{2}\right)$ as a part of a set union, so the frequency of $s_{1}$ in the union can be revealed. Hence, if all values of $\eta$ are distinct, we can learn the multi-set union.

\footnotetext{
${ }^{4}$ Note that one has to solve $\bar{\ell}$ DLPs over a group of order $q_{i}$ for one decryption in the NS encryption scheme. In Step 3 (b), one has to solve $2 d=2 n k$ DLPs over a group of order $q_{i}$ for each $q_{i}$. It requires $O\left(\sqrt{d q_{i}}\right)$ multiplications to solve $d$ DLPs over a group of order $q_{i}[18]$ and hence total complexity of this step is $O\left(\bar{\ell} \sqrt{d q_{j}}\right)$ multiplications.
} 
Consider the probability that there exist at least two same values among $d$ values of function $\eta$. This probability is

$$
1-\left(1-\frac{1}{2^{\tau \ell^{\prime \prime}}}\right) \cdots\left(1-\frac{d-1}{2^{\tau \ell^{\prime \prime}}}\right) \approx \frac{d^{2}}{2^{\tau \ell^{\prime \prime}}}
$$

and it is less than $2^{-\lambda}$ if $\ell^{\prime \prime}>\frac{\lambda+2 \log d-1}{\log d}$. For example, when $\lambda=80$ and $d \approx d_{0} \approx 2^{10}$, then $\ell^{\prime \prime}$ is about 10 .

Both computational and communicational complexities of our multi-set union protocol are the same with those of our set union protocol as big-O notation. It is heavier than the previous best result [14], which requires $O\left(n^{2} k\right)$ exponentiations in $\mathbb{F}_{q}$ and $O\left(n^{2} k \log q\right)$ bits where $q$ is similar to the size of the universe. However, the public key size of our protocol is $O(1)$ elements, while that of the previous result in [14] is $O(d)$ elements for the size $d$ of the multi-set union since their construction utilized ElGamal encryption schemes defined over an extension field $\mathbb{F}_{p^{d}}$ of extension degree $d$.

\subsection{Set Intersection Protocol}

In most private set intersection protocols based on polynomial representations and AHE schemes, it is hard to factor the resulting polynomial corresponded to the intersection of datasets. Hence, it is assumed that the decryptors who want to obtain the set intersection possess a set having the resulting set as a subset and evaluate at elements of his set to check whether each element is a root of the resulting polynomial. However, our polynomial representation provides a private set intersection protocol in which the decryptors efficiently recover the resulting set without a superset of the resulting set, except the universe, since it enables us to factor the resulting polynomial.

\section{Conclusion}

In this paper, we provided a new representation of a set by a polynomial over $\mathbb{Z}_{\sigma}$, which can be efficiently inverted by finding all the linear factors of a polynomial whose root locates in the image of our encoding function, when the factorization of $\sigma$ is public. Then we presented an efficient constant-round set union protocols, transforming our representation into a rational function and then combining it with threshold NS AHE scheme. We also extend our set union protocol to the multi-set union protocol by modifying rational function representation and consider the set intersection protocol in the case that decryptors do not possess a superset of the resulting set except the universe.

We showed that our encoding function is quite efficient on average-case, but it still requires exponential time in the degree of a polynomial to recover a set from the polynomial represented by our encoding function at worst-case although the probability of the worst-case is sufficiently small. Hence it would be interesting to construct an encoding function that enables us to recover a set in polynomial time even at worst-case.

\section{References}

1. M. Ben-Or, S. Goldwasser, and A. Wigderson. Completeness theorems for non-cryptographic fault-tolerant distributed computation (extended abstract). In J. Simon, editor, ACM Symposium on Theory of Computing (STOC) 1988, pages 1-10. ACM, 1988.

2. M. Blanton and E. Aguiar. Private and oblivious set and multiset operations. In H. Y. Youm and Y. Won, editors, ACM Symposium on Information, Compuer and Communications Security (ASIACCS) 2012, pages 40-51. ACM, 2012. 
3. J. Camenisch and V. Shoup. Practical verifiable encryption and decryption of discrete logarithms. In D. Boneh, editor, Advances in Cryptology - CRYPTO 2003, volume 2729 of LNCS, pages 126-144. Springer, 2003.

4. J. Camenisch and M. Stadler. Proof systems for general statements about discrete logarithms. Technical Report, No. 260, March 1997, 1997.

5. R. Cramer, I. Damgård, and J. B. Nielsen. Multiparty computation from threshold homomorphic encryption. In B. Pfitzmann, editor, EUROCRYPT 2001, volume 2045 of LNCS, pages 280-299. Springer, 2001.

6. E. D. Cristofaro, J. Kim, and G. Tsudik. Linear-complexity private set intersection protocols secure in malicious model. In M. Abe, editor, ASIACRYPT 2010, volume 6477 of LNCS, pages 213-231. Springer, 2010.

7. E. D. Cristofaro and G. Tsudik. Practical private set intersection protocols with linear complexity. In R. Sion, editor, Financial Cryptography (FC) 2010, volume 6052 of LNCS, pages 143-159. Springer, 2010.

8. P.-A. Fouque, G. Poupard, and J. Stern. Sharing decryption in the context of voting or lotteries. In Y. Frankel, editor, Financial Cryptography (FC) 2000, volume 1962 of LNCS, pages 90-104. Springer, 2001.

9. M. J. Freedman, K. Nissim, and B. Pinkas. Efficient private matching and set intersection. In C. Cachin and J. Camenisch, editors, EUROCRYPT 2004, volume 3027 of LNCS, pages 1-19. Springer, 2004.

10. K. B. Frikken. Privacy-preserving set union. In J. Katz and M. Yung, editors, Applied Cryptography and Network Security (ACNS) 2007, volume 4521 of LNCS, pages 237-252. Springer, 2007.

11. J. Furukawa and K. Sako. An efficient scheme for proving a shuffle. In J. Kilian, editor, CRYPTO 2001, volume 2139 of $L N C S$, pages 368-387. Springer, 2001.

12. O. Goldreich. Foundations of Cryptography: Volume I Basic Tools. Cambridge University Press, 2001.

13. O. Goldreich, S. Micali, and A. Wigderson. How to play any mental game or a completeness theorem for protocols with honest majority. In A. V. Aho, editor, ACM Symposium on Theory of Computing (STOC) 1987, pages 218-229. ACM, 1987.

14. J. Hong, J. W. Kim, J. Kim, K. Park, and J. H. Cheon. Constant-round privacy preserving multiset union. IACR Cryptology ePrint Archive, 2011. Available at http://eprint.iacr.org/2011/138.

15. S. Jarecki and X. Liu. Efficient oblivious pseudorandom function with applications to adaptive ot and secure computation of set intersection. In O. Reingold, editor, Theory of Cryptography (TCC) 2009, volume 5444 of $L N C S$, pages 577-594. Springer, 2009.

16. J. Katz and R. Ostrovsky. Round-optimal secure two-party computation. In M. K. Franklin, editor, CRYPTO 2004, volume 3152 of $L N C S$, pages 335-354. Springer, 2004.

17. L. Kissner and D. Song. Privacy-preserving set operations. In V. Shoup, editor, Advances in CryptologyCRYPTO 2005, volume 3621 of LNCS, pages 241-257, 2005.

18. F. Kuhn and R. Struik. Random walks revisited: Extensions of pollard's rho algorithm for computing multiple discrete logarithms. In S. Vaudenay and A. M. Youssef, editors, Selected Areas in Cryptography (SAC) 2001, volume 2259 of $L N C S$, pages 212-229. Springer, 2001.

19. V. K. Leont'ev. Roots of random polynomials over a finite field. Matematicheskie Zametki, 80(2):313-316, 2006.

20. P. D. MacKenzie and K. Yang. On simulation-sound trapdoor commitments. In C. Cachin and J. Camenisch, editors, EUROCRYPT, volume 3027 of LNCS, pages 382-400. Springer, 2004.

21. D. Naccache and J. Stern. A new public key cryptosystem based on higher residues. In L. Gong and M. K. Reiter, editors, ACM Conference on Computer and Communications Security (ACM CCS) 1998, pages 59-66. ACM, 1998.

22. T. Okamoto and S. Uchiyama. A new public-key cryptosystem as secure as factoring. In K. Nyberg, editor, Advances in Cryptology - EUROCRYPT 1998, volume 1403 of LNCS, pages 308-318. Springer, 1998.

23. P. Paillier. Public-key cryptosystems based on composite degree residuosity classes. In J. Stern, editor, Advances in Cryptology - EUROCRYPT 1999, volume 1592 of LNCS, pages 223-238. Springer, 1999.

24. Y. Sang and H. Shen. Efficient and secure protocols for privacy-preserving set operations. ACM Transactions on Information and System Security, 13(1), 2009.

25. J. H. Seo, J. H. Cheon, and J. Katz. Constant-round multi-party private set union using reversed Laurent series. In M. Fischlin, editor, Public Key Cryptography (PKC) 2012, LNCS. Springer, 2012. To appear.

26. A. Shamir. How to share a secret. Communications of the ACM, 22(11):612-613, 1979.

27. A. Shamir. On the generation of multivariate polynomials which are hard to factor. In S. R. Kosaraju, D. S. Johnson, and A. Aggarwal, editors, ACM Symposium on Theory of Computing (STOC) 1993, pages 796-804. ACM, 1993.

28. V. Shoup. Practical threshold signatures. In B. Preneel, editor, EUROCRYPT 2000, volume 1807 of $L N C S$, pages 207-220. Springer, 2000.

29. V. Shoup. A Computational Introduction to Number Theory and Algebra. Cambridge University Press, 2nd edition, 2009.

30. K. Suzuki, D. Tonien, K. Kurosawa, and K. Toyota. Birthday paradox for multi-collisions. In M. S. Rhee and B. Lee, editors, Information Security and Cryptography (ICISC) 2006, volume 4296 of LNCS, pages 29-40. Springer, 2006.

31. C. Umans. Fast polynomial factorization and modular composition in small characteristic. In C. Dwork, editor, ACM Symposium on Theory of Computing (STOC) 2008, pages 481-490. ACM, 2008.

32. J. von zur Gathen and J. Gerhard. Modern Computer Algebra. Cambridge University Press, 2nd edition, 1999. 


\section{A The Proper Size of $\alpha$}

In this section, we explain why the proper size of $\alpha$ is $\frac{1}{3}$. Let $\alpha=\frac{b}{a}$ for relatively prime $a, b$ with $a<b$ and assume that $\ell$ and $\ell^{\prime}$ are divided by $b$. Let $h:\{0,1\}^{*} \rightarrow\{0,1\}^{3(1-\alpha) \tau}$ and $h_{i}:\{0,1\}^{*} \rightarrow\{0,1\}^{3 \alpha \tau}$ be uniform hash functions for $1 \leq i \leq \ell^{\prime} .{ }^{5}$ Assume that $3 \alpha \tau$ and $3(1-\alpha) \tau$ are integers. Parse a message $s_{i} \in \mathcal{U} \subseteq\{0,1\}^{3 \alpha \tau \ell}$ into $\ell$ blocks $s_{i, 1}, \cdots, s_{i, \ell}$ of $3 \alpha \tau$-bit so that $s_{i}=s_{i, 1}\|\cdots\| s_{i, \ell}$. Let $s_{i, \ell+\underline{j}}=h_{j}\left(s_{i}\right)$ for $1 \leq j \leq \ell^{\prime}$ and parse $h\left(s_{j}\right)$ into $(a-b)$ blocks $s_{i, \bar{\ell}+1}, \cdots, s_{i, \bar{\ell}+a-b}$ of $3 \alpha \tau$-bit. Set $\bar{\ell}=\ell+\ell^{\prime}$.

We define an encoding function $\iota_{\alpha}: \mathcal{U} \subseteq\{0,1\}^{3 \alpha \tau \ell} \rightarrow \mathbb{Z}_{\sigma}$, in which $\iota_{\alpha}\left(s_{i}\right)$ is the unique element in $\mathbb{Z}_{\sigma}$ satisfying $\iota_{\alpha}\left(s_{i}\right)=s_{i,(j-1) b+1}\|\cdots\| s_{i,(j-1) b+a} \bmod q_{j}$ for a composite $\sigma=\prod_{j=1}^{\bar{\ell}} q_{j}$. Then a set $S$ is represented as a polynomial $f(x)=\prod_{s_{i} \in S}\left(x-\iota_{\alpha}\left(s_{i}\right)\right) \in \mathbb{Z}_{\sigma}[x]$.

For each message $s_{i}=s_{i, 1}\|\cdots\| s_{i, \ell}$, denote $\iota_{\alpha}\left(s_{i}\right) \bmod q_{j}$ by $s_{j}^{(i)}$. We generalize the definition of a linkable pair. We define $\left(s_{j}^{(i)}, s_{j+1}^{\left(i^{\prime}\right)}\right) \in \mathbb{Z}_{q_{j}} \times \mathbb{Z}_{q_{j+1}}$ to be a linkable pair if the last $3(1-\alpha) \tau$-bit of $s_{j}^{(i)}$ is equal to the first $3(1-\alpha) \tau$-bit of $s_{j+1}^{\left(i^{\prime}\right)}$. Inductively, we also define $\left(s_{1}^{\left(i_{1}\right)}, \cdots, s_{j+1}^{\left(i_{j+1}\right)}\right) \in$ $\mathbb{Z}_{q_{1}} \times \cdots \times \mathbb{Z}_{q_{j+1}}$ to be a linkable pair if $\left(s_{1}^{\left(i_{1}\right)}, \cdots, s_{j}^{\left(i_{j}\right)}\right)$ and $\left(s_{j}^{\left(i_{j}\right)}, s_{j+1}^{\left(i_{j+1}\right)}\right)$ are linkable pairs.

Let $\iota_{\alpha}\left(s_{i}\right)$ and $\iota_{\alpha}\left(s_{i^{\prime}}\right)$ be images of elememts $s_{i}$ and $s_{i^{\prime}}$ of the encoding function $\iota_{\alpha}$ with $s_{i} \neq s_{i^{\prime}}$. Assume that $s_{i}$ and $s_{i^{\prime}}$ are uniformly chosen strings from $\{0,1\}^{3 \alpha \tau \ell}$. Then,

$$
\begin{aligned}
& \operatorname{Pr}\left[\left(s_{j}^{(i)}, s_{j}^{\left(i^{\prime}\right)}\right) \text { is a linkable pair }\right] \\
& =\operatorname{Pr}\left[s_{i, j b+1}\|\cdots\| s_{i,(j-1) b+a}=s_{i^{\prime}, j b+1}\|\cdots\| s_{i^{\prime},(j-1) b+a}\right] \\
& =\frac{1}{2^{3(1-\alpha) \tau}}
\end{aligned}
$$

for a fixed $1 \leq j \leq \bar{\ell}$

At the decoding phase, when a polynomial $f(x)=\prod_{i=1}^{d}\left(x-\iota_{\alpha}\left(s_{i}\right)\right)$ is given, one computes all the roots $\left\{s_{j}^{(1)}, \cdots, s_{j}^{(d)}\right\}$ over $\mathbb{Z}_{q_{j}}$. Then for each $j$ sequentially from 1 to $\bar{\ell}-1$, we find all linkable pairs between $\left\{s_{j}^{(1)}, \cdots, s_{j}^{(d)}\right\}$ and $\left\{s_{j+1}^{(1)}, \cdots, s_{j+1}^{(d)}\right\}$ and check the hash values to find the correct $d s_{i}$ 's.

Theorem 4. Assume that $S=\left\{s_{1}, \ldots, s_{d}\right\}$ is a uniformly and randomly chosen set in the set of subsets of cardinality $d$ of the set $\{0,1\}^{3 \alpha \tau \ell}$ for $0<\alpha<1$. Define an encoding function $\iota_{\alpha}:\{0,1\}^{3 \alpha \tau \ell} \rightarrow \mathbb{Z}_{\sigma}$ so that $\iota_{\alpha}\left(s_{i}\right)$ is the unique element in $\mathbb{Z}_{\sigma}$ satisfying $\iota_{\alpha}\left(s_{i}\right) \equiv$ $s_{i,(j-1) b+1}\|\cdots\| s_{i,(j-1) b+a} \bmod q_{j}$ for all $1 \leq j \leq \bar{\ell}$ when $s_{i}=s_{i, 1}\|\cdots\| s_{i, \ell}, s_{i, j}$ 's are $3 \alpha \tau$ bit and $\alpha=\frac{b}{a}$ for relatively prime $a, b$. Assume that $\ell$ and $\ell^{\prime}$ are divided by $b$. Assume $h$ and $h_{j}$ 's utilized in the encoding function $\iota$ are uniform hash functions.

The expected number of linkable pairs of $\bar{\ell}$-tuple is at most

$$
d\left(1+\frac{3}{2^{\min \{3 \alpha \tau,(2-3 \alpha) \tau\}}}\right)^{\bar{\ell}-1}
$$

for a polynomial $f(x)=\prod_{i=1}^{d}\left(x-\iota_{\alpha}\left(s_{i}\right)\right)$.

Proof. Let $\mathrm{E}_{j}$ be the expected number of linkable pair of $j$-tuple in $\mathbb{Z}_{q_{1}} \times \cdots \times \mathbb{Z}_{q_{j}}$ for $j \geq 2$. For $1 \leq j \leq j^{\prime} \leq \bar{\ell}$, let $S_{j^{\prime}-j+1}\left(i_{j}, \ldots, i_{j^{\prime}}\right)$ be the event that $\left(s_{j}^{\left(i_{j}\right)}, \ldots, s_{j^{\prime}}^{\left(i_{j}^{\prime}\right)}\right)$ is a linkable pair.

\footnotetext{
${ }^{5}$ Note that $q_{j}$ 's are $(3 \tau+1)$-bit primes and the outputs of hash functions $h$ and $h_{i}$ 's are less than $q_{j}$ for all $j$.
} 
Then,

$$
\begin{aligned}
\mathrm{E}_{2} & =\sum_{i_{1}, i_{2} \in\{1, \ldots, d\}} 1 \cdot \operatorname{Pr}\left[\mathrm{S}_{2}\left(i_{1}, i_{2}\right)\right] \\
& =\sum_{i_{1}, i_{2} \in\{1, \ldots, d\}} \operatorname{Pr}\left[\mathrm{S}_{2}\left(i_{1}, i_{2}\right) \wedge\left(i_{1}=i_{2}\right)\right]+\sum_{i_{1}, i_{2} \in\{1, \ldots, d\}} \operatorname{Pr}\left[\mathrm{S}_{2}\left(i_{1}, i_{2}\right) \wedge\left(i_{1} \neq i_{2}\right)\right] \\
& =\sum_{i_{1} \in\{1, \ldots, d\}} \operatorname{Pr}\left[\mathrm{S}_{2}\left(i_{1}, i_{1}\right)\right]+\sum_{i_{1} \neq i_{2} \in\{1, \ldots, d\}} \operatorname{Pr}\left[\mathrm{S}_{2}\left(i_{1}, i_{2}\right)\right] \\
& =d+d(d-1) \frac{1}{2^{3 \alpha \tau}}=d\left(1+\frac{d-1}{2^{3 \alpha \tau}}\right)
\end{aligned}
$$

since $\operatorname{Pr}\left[\mathrm{S}_{2}\left(i_{1}, i_{1}\right)\right]=1$ for $i_{1} \in\{1, \ldots, d\}$ and $\operatorname{Pr}\left[\mathrm{S}_{2}\left(i_{1}, i_{2}\right)\right]=\frac{1}{2^{3 \alpha \tau}}$ for distinct $i_{1}, i_{2} \in\{1, \ldots, d\}$ from the equation (6).

Now, we consider the relation between $\mathrm{S}_{j}$ and $\mathrm{S}_{j+1}$. When $\left(s_{1}^{\left(i_{1}\right)}, \ldots, s_{j}^{\left(i_{j}\right)}\right)$ is a linkable pair, consider the case that $\left(s_{1}^{\left(i_{1}\right)}, \ldots, s_{j}^{\left(i_{j}\right)}, s_{j+1}^{\left(i_{j+1}\right)}\right)$ is a linkable pair. One can classify this case into the following cases:

1. $i_{j+1}=i_{j}$,

2. $i_{j+1}=i_{j-k} \notin\left\{i_{j-k+1}, \ldots, i_{j}\right\}$ for $k=0, \ldots,\left\lfloor\frac{a-1}{b}\right\rfloor-1$,

3. $i_{j+1}=i_{j-k} \notin\left\{i_{j-k+1}, \ldots, i_{j}\right\}$ for $k=\left\lfloor\frac{a-1}{b}\right\rfloor, \ldots, j-1$.

At the first case, if $i_{j+1}=i_{j}$ and $\left\{s_{1}^{\left(i_{1}\right)}, \cdots, s_{j}^{\left(i_{j}\right)}\right\}$ is a linkable pair, then $\left\{s_{1}^{\left(i_{1}\right)}, \cdots, s_{j}^{\left(i_{j}\right)}, s_{j+1}^{\left(i_{j+1}\right)}\right\}$ is always a linkable pair. Hence,

$$
\begin{aligned}
\mathrm{E}_{j+1}^{(1)} & :=\sum_{i_{1}, \ldots, i_{j+1} \in\{1, \ldots, d\}} \operatorname{Pr}\left[\mathrm{S}_{j+1}\left(i_{1}, \ldots, i_{j}, i_{j+1}\right) \wedge\left(i_{j+1}=i_{j}\right)\right] \\
& =\sum_{i_{1}, \ldots, i_{j} \in\{1, \ldots, d\}} \operatorname{Pr}\left[\mathrm{S}_{j}\left(i_{1}, \ldots, i_{j}\right)\right]=\mathrm{E}_{j} .
\end{aligned}
$$

At the second case, if $0 \leq k \leq\left\lfloor\frac{a-1}{b}\right\rfloor-1$,

$$
\begin{aligned}
\mathrm{E}_{j+1}^{(2)} & :=\sum_{\substack{i_{1}, \ldots, i_{j+1} \in\{1, \ldots, d\}\\
}} \operatorname{Pr}\left[\mathrm{S}_{j+1}\left(i_{1}, \ldots, i_{j+1}\right) \wedge\left(i_{j+1}=i_{j-k} \notin\left\{i_{j-k+1}, \ldots, i_{j}\right\}\right)\right] \\
& \sum_{\substack{i_{1}, \ldots, i_{j}+1 \in\{1, \ldots, d\} \\
i_{j+1}=i_{j-k} \notin\left\{i_{j-k+1}, \ldots, i_{j}\right\}}} \operatorname{Pr}\left[\mathrm{S}_{j}\left(i_{1}, \ldots, i_{j}\right) \wedge \mathrm{S}_{j}^{\prime}\left(i_{j}, i_{j+1}\right)\right] \\
& \leq \sum_{k=0}^{\left\lfloor\frac{a-1}{b}\right\rfloor-1} \sum_{i_{1}, \ldots, i_{j} \in\{1, \ldots, d\}} \frac{1}{2^{3 k \alpha \tau}} \operatorname{Pr}\left[\mathrm{S}_{j}\left(i_{1}, \ldots, i_{j}\right)\right] \leq \frac{2}{2^{3 \alpha \tau}} \mathrm{E}_{j}
\end{aligned}
$$

since the last $\tau\left(1-\frac{(k+1) b+1}{a}\right)$-bit of $s_{j-k}^{\left(i_{j-k}\right)}$ and the first $\tau\left(1-\frac{(k+1) b+1}{a}\right)$-bit of $s_{j+1}^{\left(i_{j+1}\right)}$ are already same from the encoding rule of $\iota_{\alpha}$ when $\mathrm{S}_{j}^{\prime}\left(i_{j}, i_{j+1}\right)$ is the event that $s_{i_{j},(j-k-1) b+a+1}\|\cdots\| s_{i_{j},(j-1) b+a}=$ $s_{i_{j+1},(j-k-1) b+a+1}\|\cdots\| s_{i_{j+1},(j-1) b+a}$.

For $\left\lfloor\frac{a-1}{b}\right\rfloor \leq k<j$, if $i_{j+1}=i_{j-k} \notin\left\{i_{j-k+1}, \ldots, i_{j}\right\}$, then

$$
\{(j-k) b+1, \ldots,(j-k-1) b+a)\} \cap\{j b+1, \ldots, j b+a\}=\varnothing .
$$


Hence,

$$
\begin{aligned}
\mathrm{E}_{j+1}^{(3)} & :=\sum_{\substack{i_{1}, \ldots, i_{j+1} \in\{1, \ldots, d\}\\
}} \operatorname{Pr}\left[\mathrm{S}_{j+1}\left(i_{1}, \ldots, i_{j+1}\right) \wedge\left(i_{j+1}=i_{j-k} \notin\left\{i_{j-k+1}, \ldots, i_{j}\right\}\right)\right] \\
& \sum_{\substack{i_{1}, \ldots, i_{j+1} \in\{1, \ldots, d\} \\
i_{j+1}=i_{j-k} \notin\left\{i_{j-k+1}, \ldots, i_{j}\right\}}} \operatorname{Pr}\left[\mathrm{S}_{j}\left(i_{1}, \ldots, i_{j}\right)\right] \cdot \operatorname{Pr}\left[\mathrm{S}_{2}\left(i_{j}, i_{j+1}\right)\right] \\
& =\sum_{k=\left\lceil\frac{a-1}{b}\right\rceil}^{j-1} \sum_{i_{1}, \ldots, i_{j} \in\{1, \ldots, d\}} \frac{1}{2^{3(1-\alpha) \tau}} \operatorname{Pr}\left[\mathrm{S}_{j}\left(i_{1}, \ldots, i_{j}\right)\right] \leq \frac{d}{2^{3(1-\alpha) \tau}} \mathrm{E}_{j} .
\end{aligned}
$$

since $\sum_{k=\left\lceil\frac{a-1}{b}\right\rceil}^{j-1} \frac{1}{2^{3(1-\alpha) \tau}} \leq \frac{\bar{\ell}}{2^{3(1-\alpha) \tau}} \leq \frac{d}{2^{3(1-\alpha) \tau}}$.

From the above results, one can obtain the recurrence formula of $\mathrm{E}_{j}$ as follows:

$$
\begin{aligned}
\mathrm{E}_{j+1} & =\mathrm{E}_{j+1}^{(1)}+\mathrm{E}_{j+1}^{(2)}+\mathrm{E}_{j+1}^{(3)} \\
& \leq\left(1+\frac{2}{2^{3 \alpha \tau}}+\frac{d}{2^{3(1-\alpha) \tau}}\right) \mathrm{E}_{j}
\end{aligned}
$$

for $j \geq 2$. Therefore,

$$
\begin{aligned}
\mathrm{E}_{\bar{\ell}} & \leq\left(1+\frac{2}{2^{3 \alpha \tau}}+\frac{d}{2^{3(1-\alpha) \tau}}\right) \sum_{i_{1}, \ldots, i_{\bar{\ell}-1} \in\{1, \ldots, d\}} \operatorname{Pr}\left[\mathrm{S}_{\bar{\ell}}\left(i_{1}, \ldots, i_{\bar{\ell}-1}\right)\right] \\
& \leq\left(1+\frac{2}{2^{3 \alpha \tau}}+\frac{d}{2^{3(1-\alpha) \tau}}\right)^{\bar{\ell}-2} \sum_{i_{1}, i_{2} \in\{1, \ldots, d\}} \operatorname{Pr}\left[\mathrm{S}_{2}\left(i_{1}, i_{2}\right)\right] \\
& \leq d\left(1+\frac{3}{2^{\min \{3 \alpha \tau,(2-3 \alpha) \tau\}}}\right)^{\bar{\ell}-1} .
\end{aligned}
$$

If $\bar{\ell} \geq 2^{\min \{3 \alpha \tau,(2-3 \alpha) \tau\}}$, then $d\left(1+\frac{3}{2^{\min \{3 \alpha \tau,(2-3 \alpha) \tau\}}}\right)^{\bar{\ell}-1}$ is exponentially increased. Hence it requires the limitation of $\bar{\ell}$ so that $\bar{\ell}<2^{\min \{3 \alpha \tau,(2-3 \alpha) \tau\}}$. But, if $\alpha \neq \frac{1}{3}$, then either $3 \alpha$ or $(2-3 \alpha)$ is less than 1 and hence $2^{\min \{3 \alpha \tau,(2-3 \alpha) \tau\}}<\min \left\{d, \frac{\lfloor\log N\rfloor-2}{3 \tau}\right\}$ for increasing of $d$ and $\log N$. It causes the limitation of the size of universe since $|\mathcal{U}| \leq 2^{3 \alpha \tau \ell}$ in the case $\alpha \neq \frac{1}{3}$. From these reasons, we set $\alpha$ so that the universe $\mathcal{U}$ is a subset of $\{0,1\}^{\tau \ell}$, i.e., $\alpha=\frac{1}{3}$.

\section{B Threshold Naccache-Stern Cryptosystem}

In this section, we provide a threshold version of NS AHE scheme. Our threshold version is based on Fouque et al.'s work [8], which transforms the original Paillier homomorphic encryption scheme into a threshold version working from Shoup's technique [28]. The security proof of our construction is similar with that of a threshold version of Paillier encryption scheme under the assumption that the original NS AHE scheme is semantically secure. We omit the detail of the security proof.

Threshold Naccache-Stern Encryption Scheme Our threshold version of the NS AHE scheme consists of the following four algorithms:

- KeyGen $(\lambda, n, t)$ : this algorithm is executed by a dealer. It takes as inputs a security parameter $\lambda$, the number $n$ of participating users and the threshold parameter $t$. Then the dealer runs the following steps: 
1. Generate $\bar{\ell}(\tau(\lambda)+1)$-bit primes $q_{i}$ 's where $\tau(\lambda)$ is a polynomial in $\lambda$. We simply write $\tau(\lambda)$ by $\tau$. Let $u=\prod_{i=1}^{\bar{\ell} / 2} q_{i}$ and $v=\prod_{i=\bar{\ell} / 2+1}^{\bar{\ell}} q_{i}$.

2. Generate two $\kappa(\lambda)$-bit large primes $p_{1}^{\prime}, p_{2}^{\prime}$.

3. Compute $p_{1}=2 u p_{1}^{\prime}+1$ and $p_{2}=2 v p_{2}^{\prime}+1$. If at least one of $p_{1}$ and $p_{2}$ are not prime, go to the first step. Otherwise, let $N=p_{1} p_{2}$ and $\sigma=u v$.

4. The secret key $\phi(N) / \sigma$ is shared using the secret sharing scheme such as Shamir's [26]: Let $f(x)=\sum_{i=0}^{t} a_{i} x^{i}$ where $a_{0}=\phi(N) / \sigma$ and $a_{i}$ 's are randomly chosen element in $\mathbb{Z}_{\phi(N)}$. Then the secret share $\mathrm{sk}_{i}$ for a user $\mathcal{P}_{i}$ is $f(i) \bmod \phi(N) / \sigma$.

5. Choose a random element $g$ of order $\phi(N) / 4$ in $\mathbb{Z}_{N}^{*}$.

6. Choose a random generator vk of the maximal cyclic group consisting of squares in $\mathbb{Z}_{N}^{*}$. Let $\mathrm{vk}_{i}=\mathrm{vk}^{\Delta \mathrm{sk}_{i}} \bmod N$ where $\Delta=n !$.

It outputs a public key pk $=\left(N, g, \sigma, \mathrm{vk},\left\{\mathrm{vk}_{i}\right\}_{i=1}^{n}, \Delta\right)$ and a secret key sk ${ }_{i}$ for each user $\mathcal{P}_{i}$.

- Enc $(\mathrm{pk}, m)$ : this algorithm takes as inputs a public key pk and a message $m$. It chooses a random element $x$ in $\mathbb{Z}_{N}$ and outputs $c=g^{m} x^{\sigma} \bmod N$.

- ShareDec $\left(\mathrm{pk}, i, \mathrm{sk}_{i}, c\right)$ : this algorithm takes as inputs a public key pk, an index $1 \leq i \leq n$, a secret share $\mathrm{sk}_{i}$ and a ciphertext $c$. Then it computes $c_{i}=c^{2 \Delta \mathrm{sk}_{i}}$ along with its proof $\pi_{c_{i}}$ of the equality of the discrete logarithm between $\left(c^{4}, c_{i}^{2}\right)$ and $\left(\mathbf{v k}, \mathbf{v k}_{i}\right)$ and computes $g_{i}=g^{\Delta \mathbf{s k}_{i}}$ along with its proof $\pi_{g_{i}}$ of the equality of the discrete logarithm between $\left(g, g_{i}\right)$ and $\left(\mathbf{v k}, \mathbf{v k}_{i}\right)$ where $g_{i}=g^{\Delta \mathbf{s k}_{i}}$ and $\mathbf{v k}_{i}=\mathrm{vk}^{\Delta \mathrm{sk}_{i}}$. It outputs $\left(c_{i}, \pi_{c_{i}}, g_{i}, \pi_{g_{i}}\right)$.

- Combine(pk, $S, c,\left\{c_{i}\right\}_{i \in S},\left\{\pi_{c_{i}}\right\}_{i \in S},\left\{g_{i}\right\}_{i \in S},\left\{\pi_{g_{i}}\right\}_{i \in S}$ ): this algorithm is executed by the combiner. (Note that it can be also executed by any user if the fairness is not considered.) It takes as an input a public key pk, an index set $S$, a ciphertext $c$, its decryption shares $\left\{c_{i}\right\}_{i \in S}$, corresponding proofs $\left\{\pi_{c_{i}}\right\}_{i \in S}$, generator shares $\left\{g_{i}\right\}_{i \in S}$, and corresponding proofs $\left\{\pi_{g_{i}}\right\}_{i \in S}$. Then it runs the following steps:

1. If $|S| \leq t$, this algorithm returns $\perp$.

2. Otherwise, it verifies all proofs $\pi_{c_{i}}$ 's and $\pi_{g_{i}}$ 's. If at least one of proofs are failed to verify, return $\perp$.

3. For a set $S$, compute $\mu_{0, j}^{S}:=\Delta \prod_{j^{\prime} \in S \backslash\{j\}} \frac{j^{\prime}}{j^{\prime}-j} \in \mathbb{Z}$. Then compute $\bar{c}=\prod_{j \in S} c_{j}^{2 \mu_{0, j}^{S}} \bmod N$ and $\bar{g}=\prod_{j \in S} g_{j}^{2 \mu_{0, j}^{S}} \bmod N$.

4. For all $q_{i} \mid \sigma$, compute $m_{i}^{\prime}=\log _{\bar{g}} \hat{q}_{i} \hat{c}^{\hat{q_{i}}}$ using a DLP solving algorithm where $\hat{q}_{i}=\frac{\sigma}{q_{i}}$. Then compute $m^{\prime}$ such that $m^{\prime} \equiv m_{i}^{\prime} \bmod q_{i}$ for all $i$ using CRT computation. It outputs $m^{\prime}$.

Correctness of Our Construction By the Lagrange interpolation formula, we obtain

$$
\bar{c}=\prod_{j \in S} c_{j}^{2 \mu_{0, j}^{S}}=\prod_{j \in S} c^{4 \Delta \mathbf{s k}_{i} \mu_{0, j}^{S}}=c^{4 \Delta \sum_{j \in S} \mathbf{s k}_{j} \mu_{0, j}^{S}}=c^{4 \Delta^{2} f(0)}
$$

and similarly $\bar{g}=g^{4 \Delta^{2} f(0)}$. Hence,

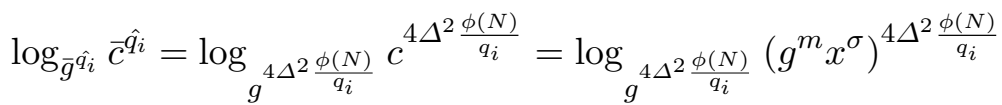

$$
\begin{aligned}
& =\log _{g}{ }_{g} \frac{\phi(N)}{q_{i}}\left(g^{m}\right)^{4 \Delta^{2} \frac{\phi(N)}{q_{i}}} \equiv\left(m \bmod q_{i}\right)
\end{aligned}
$$

which gives the correctness of our construction.

\section{Supplement for Our Set Union Protocol}

\section{C.1 Malicious Case}

Zero-knowledge Proofs We exploit the following zero-knowledge proofs for the malicious adversary model. We can efficiently construct the required zero-knowledge proofs for the NS 
encryption scheme by applying some standard techniques $[4,5]$. We briefly introduce how to construct the following zero-knowledge proofs. Let $\mathcal{E}_{\mathrm{pk}}$ be an encryption of a polynomial defined in Section 2.3.

- ZKPK $\left[g(x) \mid \mathcal{E}_{\mathrm{pk}}(g(x)), \mathcal{E}_{\mathrm{pk}}(f(x)), \mathcal{E}_{\mathrm{pk}}(f(x) \cdot g(x))\right]$ : this is a zero-knowledge proof that $\mathcal{E}_{\mathrm{pk}}(f(x)$. $g(x))$ is an encryption of $f(x) g(x)$ when polynomial encryptions $\mathcal{E}_{\mathrm{pk}}(g(x)), \mathcal{E}_{\mathrm{pk}}(f(x))$ and $\mathcal{E}_{\mathrm{pk}}(f(x) \cdot g(x))$ are given. In this case, the prover knows only $g(x)$, not $f(x)$. We obtain this protocol by generalizing zero-knowledge proof of correct multiplication which proves $\operatorname{Enc}_{\mathrm{pk}}(c)$ is an encryption of $a b$, when $\operatorname{Enc}_{\mathrm{pk}}(a)$ and $\operatorname{Enc}_{\mathrm{pk}}(b)$ are given for an AHE scheme Enc $_{\mathrm{pk}}$ with the public key pk. This protocol requires $O\left(n k^{2}\right)$ exponentiations for computation and $O\left(n k^{2}\right)(\log N)$-bit elements for communication when $f(x)$ is a polynomial of degree $2 n k$ and $g(x)$ is a polynomial of degree $k$.

- ZKPK $f f(x), g(x)]$ : this is a zero-knowledge proof that $g(x)$ is the RLS representation of $1 / f(x)$ when encryptions of $f(x)$ and $g(x)$ are given. By the Lemma 2 in [25], if $f(x)$ and $g(x)$ satisfy $\operatorname{deg}\left(f(x) g(x)-x^{(\operatorname{deg} f+\operatorname{deg} g)}\right)<\operatorname{deg} f$, then $g(x)$ is the RLS representation of a rational function $1 / f(x)$. Hence it is enough to prove that the $\operatorname{deg}(g(x))+1$ higherorder coefficients of $f(x) g(x)$ are equal to $1,0, \ldots, 0$. To prove this, the prover first gives $\mathcal{E}_{\mathrm{pk}}(f(x) \cdot g(x))$ with zero-knowledge proof ZKPK $\left[g(x) \mid \mathcal{E}_{\mathrm{pk}}(g(x)), \mathcal{E}_{\mathrm{pk}}(f(x)), \mathcal{E}_{\mathrm{pk}}(f(x) \cdot g(x))\right]$. (In this case, the prover also knows $f(x)$, but the protocol is the same.) Then, using zeroknowledge protocols that a ciphertext is an encryption of 0 and a ciphertext is an encryption of 1 , the prover proves that the encryption of the $(\operatorname{deg} g)+1$ higher-order coefficients of $\mathcal{E}_{\mathrm{pk}}(f(x) \cdot g(x))$ are encryption of $1,0, \ldots, 0$. It requires $O\left(n k^{2}\right)$ exponentiations and $O\left(n k^{2}\right)$ $(\log N)$-bit elements for communications when $f(x)$ is a polynomial of degree $k$ and $g(x)$ is a polynomial of degree $2 n k$.

Commitment Scheme We also exploit some equivocal commitment schemes [16,20] so that the simulator in the malicious adversary model can open the envelope to arbitrary value without being detected by the adversary.

Our Set Union Protocol for Malicious Case We give a PPSU-MAL protocol which is secure against malicious adversaries in Figure 4. The parameters are the same with that of protocols for the HBC adversaries model in Section 4.2.

This protocol also runs in $O(1)$ round. The complexities are the same with the protocol for the HBC adversary model as a big-O notation except those of running zero-knowledge proof protocols. However, to give a zero-knowledge proof of polynomial multiplication and inverse relation, we need to $O\left(n k^{2}\right)$ communication cost and $O\left(n k^{2}\right)$ computational cost. In particular, a zero-knowledge proof protocol ZKPK $\left[r_{i, j} \mid \Lambda\left(r_{i, j}\right), \mathcal{E}_{\mathrm{pk}}\left(F_{S_{i, j}}\right), \mu_{i, j}\right]$ has to run for all $1 \leq i \neq j \leq n$, the total communication complexity and computational complexity are $O\left(n^{3} k^{2}\right)(\log N)$-bit elements and $O\left(n^{3} k^{2}\right)$ exponentiation, respectively, and this is the most expensive part in our malicious protocol.

The correctness is similar with that of the HBC case and the following theorem guarantees the security of the protocol proposed in Figure 4.

Theorem 5. In our set union protocol for the malicious case described in Figure 4, there is a simulator $\mathcal{S}$ for a player (or a group of players) operating in the ideal model, such that the view of the players in the ideal model is computationally indistinguishable from the view of the honest players and any adversaries $\mathcal{A}$ of colluding players in the real world.

Proof. Let $\mathcal{S}$ be a simulator in ideal world, communicating with malicious adversaries $\mathcal{A}$ in the real world, who are able to collude among malicious adversaries. We want to show that malicious adversaries $\mathcal{A}$ in the real world cannot distinguish that $\mathcal{S}$ does not play in the real world.

$\mathcal{S}$ operates the following steps: 
Input: There are $n \geq 2$ participants $\mathcal{P}_{i}$ with a private input set $S_{i} \subseteq \mathcal{U}$ of cardinality $k$. Set $d=n k$. The participants share the secret key sk, to which pk is the corresponding public key to the threshold NS AHE scheme. Let $\mathcal{E}_{\mathrm{pk}}$ be an encryption defined in Section 2.3. Let $\iota:\{0,1\}^{*} \rightarrow \mathbb{Z}_{\sigma}$ be the encoding function provided in Section 3. We utilize an equivocal commitment scheme and zero-knowledge proofs protocols.

Each participant $\mathcal{P}_{i}, i=1, \ldots, n$ :

1. (a) constructs the polynomial $f_{S_{i}}(x)=\prod_{s_{i, j} \in S_{i}}\left(x-\iota\left(s_{i, j}\right)\right) \in \mathbb{Z}_{\sigma}[x]$, runs RationalToRLS $\left(1, f_{S_{i}},(2 n+1) k-1\right)$ to obtain $\left(\frac{1}{f_{S_{i}}(x)}\right)_{[-(2 n+1) k+1,-k]}$, and computes $F_{S_{i}}(x)=\left(\frac{1}{f_{S_{i}}(x)}\right)_{[-(2 n+1) k+1,-k]} \cdot x^{(2 n+1) k-1}$.

(b) computes $\tilde{f_{S_{i}}}, \tilde{F}_{S_{i}}$, the encrypted polynomial of $f_{S_{i}}, F_{S_{i}}$ and sends them to all other participants with proofs of ZKPK $\left[f_{S_{i}}, F_{S_{i}}\right]$.

(c) chooses random polynomials $r_{i, j}(x)$ of degree at most $k$ for all $1 \leq j \leq n$, and sends a commitment of $\Lambda\left(r_{i, j}\right)$ to all parties, where $\Lambda\left(r_{i, j}\right)=\mathcal{E}_{p k}\left(r_{i, j}\right)$.

2. (a) opens the commitment to $\Lambda\left(r_{i, j}\right)$.

(b) verifies zero-knowledge proofs $\operatorname{ZKPK}\left[f_{S_{i}}, F_{S_{i}}\right]$.

(c) sets the leading coefficient to a known encryption of 1

(d) calculates $\mu_{i, j}$, the encrypted polynomial of $F_{S_{j}} \times r_{i, j}$ with proofs of correct multiplication $\operatorname{ZKPK}\left[r_{i, j} \mid \Lambda\left(r_{i, j}\right), \mathcal{E}_{\mathrm{pk}}\left(F_{S_{i, j}}\right), \mu_{i, j}\right]$ and sends them all other participants.

3. (a) calculates the encrypted polynomial of $F(x)=\sum_{i=1}^{n} \sum_{j=1}^{n} F_{S_{j}} \times r_{i, j}$ and verifies all attached proofs.

(b) performs a group decryption with all other players to obtain the $2 n k$ high-order terms of $F(x)$.

4. (a) recovers a polynomial pair of $u_{j}(x)$ and $U_{j}(x)$ in $\mathbb{Z}_{q_{j}}[x]$ for all $1 \leq j \leq \bar{\ell}$ such that $\left(\frac{u_{j}(x)}{U_{j}(x)}\right)_{[-2 n k,-1]}=\left(F(x) \bmod q_{j}\right)_{[k-1,(2 n+1) k-2]} \cdot x^{-(2 n+1) k+1}$ and $\operatorname{gcd}\left(u_{j}(x), U_{j}(x)\right)=1$ in $\mathbb{Z}_{q_{j}}[x]$, using the $2 n k$ high-order terms of $F(x)$ obtained in Step 3 (b).

(b) extracts all roots of $U_{j}(x)$ in $\mathbb{Z}_{q_{j}}[x]$ for all $j$ using a factorization algorithm.

(c) determines the set union using the encoding rule of $\iota$.

Fig. 4. PPSU-MAL protocol in the malicious case

1. For each honest player $i$, a simulator $\mathcal{S}$

(a) chooses monic polynomial $f_{i}$ such that each such polynomial is relatively prime.

(b) chooses polynomials $r_{i, 1}, \ldots, r_{i, n}$ and creates encryptions $\Lambda\left(r_{i, j}\right)$ from them.

2. The simulator $\mathcal{S}$ performs Step 1 (a), (b), (c) of our set union protocol:

(a) calculates $F_{S_{i}}(x)=\left(\frac{1}{f_{S_{i}}(x)}\right)_{[-(2 n+1) k+1,-k]} \cdot x^{(2 n+1) k-1}$.

(b) computes $\tilde{f}_{S_{i}}, \tilde{F}_{S_{i}}$, the encrypted polynomial of $f_{S_{i}}, F_{S_{i}}$ and sends them to all other participants with proofs of ZKPK $\left[f_{S_{i}}, F_{S_{i}}\right]$.

(c) chooses random polynomials $r_{i, j}(x)$ of degree at most $k$ for all $1 \leq j \leq n$, and sends trapdoor commitment of $\Lambda\left(r_{i, j}\right)$ to all parties, where $\Lambda\left(r_{i, j}\right)=\mathcal{E}_{p k}\left(r_{i, j}\right)$.

(d) receives from each malicious player $\alpha \in \mathcal{A}$ the followings: $f_{S_{\alpha}}, \tilde{F}_{S_{\alpha}}, \operatorname{ZKPK}\left[f_{S_{\alpha}}, F_{S_{\alpha}}\right]$ and trapdoor commitment of $\Lambda\left(r_{\alpha, j}\right)$ for $1 \leq j \leq n$.

3. The simulator $\mathcal{S}$ extracts $f_{S_{\alpha}}$ from $\operatorname{ZKPK}\left[f_{S_{\alpha}}, F_{S_{\alpha}}\right]$ and trapdoor commitments to $\Lambda\left(r_{\alpha, j}\right)$ to obtain a polynomial $F_{\alpha}$ and polynomials $r_{\alpha, j}$ for all $\alpha \in \mathcal{A}$.

4. The simulator $\mathcal{S}$ submits all roots to TTP and returns the set union.

5. The simulator $\mathcal{S}$ prepares to reveal the set union:

(a) computes $U(x)=: \prod_{s_{j} \in U}\left(x-\iota\left(s_{j}\right)\right) \in \mathbb{Z}_{\sigma}[x]$ and computes the high-order $2 n k$ RLS representation $F(x)$ of a rational function $\frac{u(x)}{U(x)}$ over $\mathbb{Z}_{\sigma}$ for a randomly chosen $u(x)$ such that $\operatorname{gcd}(u(x), U(x))=1$ and $\operatorname{deg} u<\operatorname{deg} U$.

(b) computes $\left(F_{S_{i}}\right)_{j}:=\frac{u_{j}(x)}{U_{j}(x)} \bmod q_{j}$ where $U_{j}(x):=\prod_{s_{j} \in U}\left(x-\iota\left(s_{j}\right)\right) \bmod q_{j}$.

(c) chooses a set of polynomial $r_{i, j}$ 's such that $F(x)=\sum_{i=1}^{n} F_{S_{i}}(x)\left(\sum_{j=1}^{n} r_{i, j}\right) \in \mathbb{Z}_{\sigma}[x]$.

6. The simulator $\mathcal{S}$ follows the rest of the protocol as described and he opens the trapdoor commitment to reveal an appropriate $\Lambda\left(r_{i, j}\right)$ for the new chosen polynomial $r_{i, j}$. Then the participants calculate an encryption of the polynomial $U$ chosen by the simulator $\mathcal{S}$, and then decrypt it and hence learn the union set. 
Note that the colluding players $\mathcal{A}$ cannot distinguish that the simulator $\mathcal{S}$ is in the real world or not and all players obtain the correct answer in both the real world and ideal world. 